Florida International University FIU Digital Commons

3-24-2014

\title{
Fiber Pathways for Language in the Developing Brain: A Diffusion Tensor Imaging (DTI) Study
}

Iris J. Broce

Florida International University, ibroce@fiu.edu

DOI: $10.25148 /$ etd.FI14040891

Follow this and additional works at: https://digitalcommons.fiu.edu/etd

Part of the Biological Psychology Commons, Child Psychology Commons, Cognitive Neuroscience Commons, Developmental Neuroscience Commons, Developmental Psychology Commons, and the Systems Neuroscience Commons

\section{Recommended Citation}

Broce, Iris J., "Fiber Pathways for Language in the Developing Brain: A Diffusion Tensor Imaging (DTI) Study" (2014). FIU Electronic Theses and Dissertations. 1220.

https://digitalcommons.fiu.edu/etd/1220 


\title{
FLORIDA INTERNATIONAL UNIVERSITY
}

Miami, Florida

\section{FIBER PATHWAYS FOR LANGUAGE IN THE DEVELOPING BRAIN: A DIFFUSION TENSOR IMAGING (DTI) STUDY}

\author{
A thesis submitted in partial fulfillment of \\ the requirements for the degree of \\ MASTER OF SCIENCE \\ in \\ PSYCHOLOGY
}

by

Iris Broce 
To: Dean Kenneth G. Furton

College of Arts and Sciences

This thesis, written by Iris Broce, and entitled Fiber Pathways for Language in the Developing Brain: A Diffusion Tensor Imaging (DTI) Study, having been approved in respect to style and intellectual content, is referred to you for judgment.

We have read this thesis and recommend that it be approved.

Lorraine Bahrick

Robert Lickliter

Anthony Dick, Major Professor

Date of Defense: March 24, 2014

The thesis of Iris Broce is approved.

Dean Kenneth G. Furton

College of Arts and Sciences

Dean Lakshmi N. Reddi University Graduate School

Florida International University, 2014 


\section{ACKNOWLEDGMENTS}

I wish to acknowledge Dr. Anthony Dick, my major professor, for providing me outstanding training, fostering ideas, and continuous support throughout this journey. Thank you for your patience, guidance, and encouragement. I would also like to acknowledge my committee members, Dr. Lorraine Bahrick and Dr. Robert Lickliter for providing me insightful comments throughout the development, execution, and completion of my thesis project. In addition, I would like to acknowledge several others for their guidance and enthusiasm in my work- Dr. Byron Bernal, Dr. Magno Guillen, Dr. Margaret Friend, Dr. Amy Pace, and Dr. Leslie Carver. Finally, I wish to thank Rosalie Odean, Carla Abad, Brittany Yusko, for their good humor and friendship, all the members of the Developmental Cognitive Neuroscience Lab for their contributions to this project, and my family for their unconditional love and support throughout my academic journey. 


\title{
ABSTRACT OF THE THESIS \\ FIBER PATHWAYS FOR LANGUAGE IN THE DEVELOPING BRAIN: A DIFFUSION TENSOR IMAGING (DTI) STUDY
}

\author{
by \\ Iris Broce \\ Florida International University, 2014 \\ Miami, Florida \\ Professor Anthony Dick, Major Professor
}

The present study characterized two fiber pathways important for language, the superior longitudinal fasciculus/arcuate fasciculus (SLF/AF) and the frontal aslant tract (FAT), and related these tracts to speech, language, and literacy skill in children five to eight years old. We used Diffusion Tensor Imaging (DTI) to characterize the fiber pathways and administered several language assessments. The FAT was identified for the first time in children. Results showed no age-related change in integrity of the FAT, but did show age-related change in the left (but not right) SLF/AF. Moreover, only the integrity of the right FAT was related to phonology but not audiovisual speech perception, articulation, language, or literacy. Both the left and right SLF/AF related to language measures, specifically receptive and expressive language, and language content. These findings are important for understanding the neurobiology of language in the developing brain, and can be incorporated within contemporary dorsal-ventral-motor models for language. 


\section{TABLE OF CONTENTS}

CHAPTER

PAGE

I.INTRODUCTION

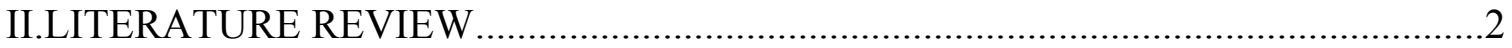

Investigating White Matter Development in vivo .............................................2

White Matter Development.................................................................................4

Contemporary Model of the Neurobiology of Language .....................................

Fiber Pathways for Speech Language, and Literacy.......................................... 8

Behavioral and Neural Components of Speech, Language, and Literacy .............10

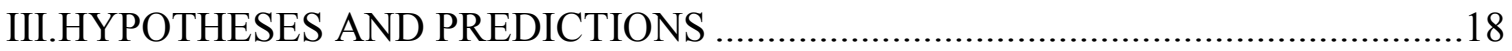

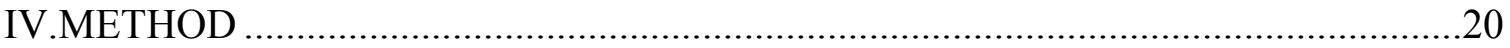

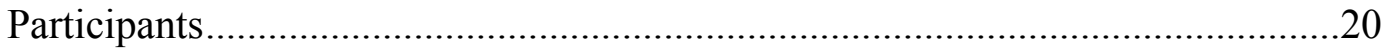

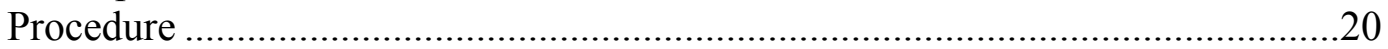

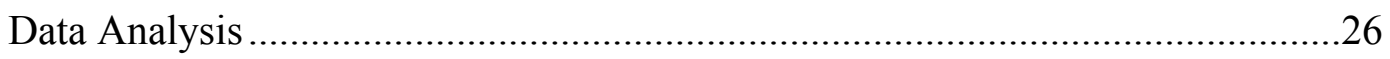

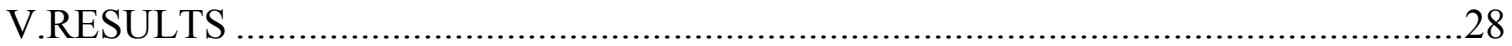

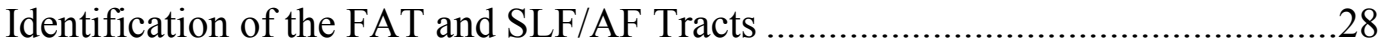

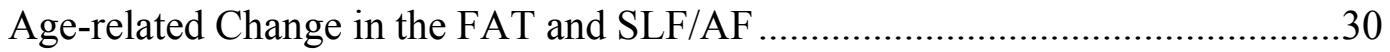

Relation of the FAT and SLF/AF to Speech, Language, and Literacy..................32

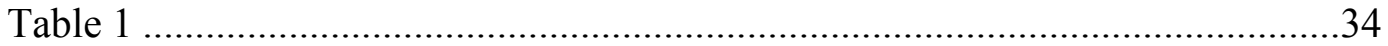

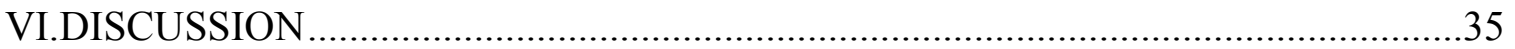

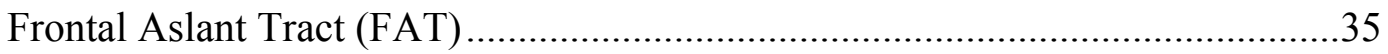

Superior Longitudinal Fasciculus/Arcuate Fasciculus (SLF/AF) .........................38

Importance of Findings for a Model of the Neurobiology of Language................40

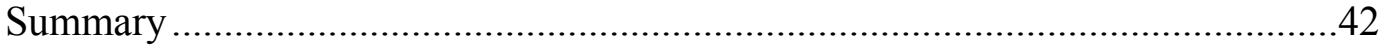

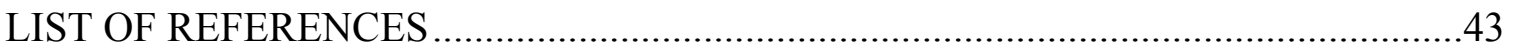




\section{LIST OF FIGURES}

FIGURE

PAGE

1. Lateral view of the left hemisphere showing the most prominent fiber pathways for language. The current study focuses on two: the SLF/AF in green and the FAT in purple. Reprinted from Dick and Small, in press.

2. Connections of the SLF/AF in lateral view.

3. Connections of the frontal aslant tract (FAT) in coronal section, with outline of the inferior frontal and superior frontal origins and terminations in the medial and lateral sagittal views. IFGOp = inferior frontal gyrus, pars opercularis; SFG $=$ superior frontal gyrus; $\mathrm{SMA}=$ supplementary motor area; Pre-SMA = presupplementary motor area.

4. Connections of the Frontal Aslant Tract (FAT) in 19 children. Note: This mosaic is displayed in radiological orientation. Therefore, the right side of the image represents the left cerebral hemisphere.

5. Left: FAT of one child was not identified in the right hemisphere under more stringent step size (of $1 \mathrm{~mm}$ ). Right: FAT appeared when the step size was adjusted to a more liberal level (of $2 \mathrm{~mm}$ ). Note: Figures are displayed in radiological orientation.

6. Left and right connections of the SLF/AF in one child.

7. Top: Age-related changes in fractional anisotropy in the left and right Frontal Aslant Tract. Bottom: Age-related changes in fractional anisotropy in the left and right Superior Longitudinal Fasciculus/Arcuate Fasciculus.

8. Top: Age-related changes in apparent diffusion coefficient in the left and right Frontal Aslant Tract. Bottom: Age-related changes in apparent diffusion coefficient in the left and right Superior Longitudinal Fasciculus/Arcuate Fasciculus 


\section{CHAPTER I}

\section{INTRODUCTION}

Although great strides have been made in understanding brain development over the last century, the relation between brain development and behavior is an active field of inquiry. Particularly, with the advent of the magnetic resonance imaging (MRI) technique of diffusion-weighted imaging, scientific research has shifted to studying white matter development, which is the development of connections between neurons in the brain and central nervous system. Using diffusion weighted imaging, white matter development can be investigated in typically developing children and related to behavior. Specifically, different white matter pathways can be identified, tracked, characterized, and quantified in terms of their structural integrity, and these metrics can be related to a number of behavioral outcome measures. In the present study we focus on identifying a new fiber pathway — the frontal aslant tract (FAT) — for the first time in young children. We then relate the putative language pathway and another pathway of the dorsal speech stream, the superior longitudinal fasciculus/arcuate fasciculus (SLF/AF), to speech, language, and literacy outcomes. The findings we report contribute to a growing understanding of brain development and its relation to behavior. 


\section{CHAPTER II}

\section{LITERATURE REVIEW}

\section{Investigating White Matter Development in vivo}

Neurons are the major components of information processing and storage in the human brain. However, they have different parts and each part contributes uniquely to neural transmission. For example, in addition to the neural cell body or soma, parts of the neuron such as dendrites or even the cell body itself, are specifically designed to facilitate the receipt of information to the neuron from other neurons (Lenroot \& Giedd, 2006). In most cases neurons also have a single axon that emanates from the neural cell body (Lenroot \& Giedd, 2006). The axon is primarily involved in transmission of information away from the neuron to other neurons. In the nervous system, these axons form tightly organized cords, much like an electrical wiring system, called white matter tracts (Lenroot \& Giedd, 2006). Through this organization, they connect different areas of the brain, sometimes several centimeters apart. Also like an electrical wiring system, most central nervous system axons are insulated. In the brain, a substance made mostly of lipids called myelin accomplishes this insulation. Myelin serves a number of functions, which contribute to a substantial increase in the efficiency and speed of neural transmission of information (Lenroot \& Giedd, 2006). Because it is a lipid substance, myelin also gives the brain's white matter its light appearance.

It has been known for more than a century that over the course of development, different parts of the brain myelinate at different rates (Flechsig, 1896), which means they acquire the full myelin sheath at different times depending on what part of the brain the axon is projecting from and to. However, historically these studies required 
postmortem examination of fetal, infant, or child brain tissue, which is prohibitive for most researchers interested in brain development. New technology call diffusionweighted imaging, or diffusion tensor magnetic resonance imaging (DTI-MRI), invented in 1994 (Basser, Mattiello, \& LeBihan, 1994), now allows the examination of the development of white matter in vivo.

Diffusion Tensor Imaging takes advantage of differences in water diffusion in axons of high versus low structural integrity. The diffusion process follows Brownian motion--that is, the random thermal motion of molecules in biological tissues. Much like a plumbing pipe, diffusion in axons, especially myelinated axons of the white matter, is anisotropic (or, directional), with water molecules moving faster along the length of an axon than perpendicular to it (Mori \& Zhang, 2006). The advances of DTI make it possible to quantify individual differences in white matter integrity (fractional anisotropy; FA) and the degree of diffusion of water molecules through different tissues (apparent diffusion coefficient; ADC). For these measures, FA takes on values of 0 (i.e., when diffusion is completely isotropic) to 1 (i.e., when diffusion is anisotropic). The ADC values range from $0.60-1.05 \times 10^{-3} \mathrm{~mm}^{2} / \mathrm{s}$, and have an identifiable range of values in typically developing brains of $0.84+/-0.11 \times 10^{-3} \mathrm{~mm}^{2} / \mathrm{s}$ (Sener, 2001). However, these values are age and region specific, which means different white matter tracts mature at different rates over development. The ADC values in deep white matter for infants 3-4 months of age are higher $\left(\mathrm{M}=1.11+/-1.20 \times 10^{-5} \mathrm{~mm}^{2} / \mathrm{s}\right)$ compared to adults (Morriss, Zimmerman, Bilaniuk, Hunter, \& Haselgrove, 1999), but ADC values decrease (at a slower rate after 4 months), reaching adult values by around 36 months (Morriss et al., 1999; Schneider, Ilyasov, Hennig, \& Martin, 2004). 
Using DTI, visualization of white matter tracts in three-dimensional space can be achieved using methods that follow the path of greatest diffusion along the $\mathrm{x}, \mathrm{y}, \mathrm{z}$ axis (Basser et al., 1994). For example, the method of tract tracing used in this study follows the value of FA from pixel to pixel to trace out fiber tracts. Advances in DTI have enriched our understanding about how particular fiber pathways connect regions to support speech, language and literacy in adults (Bernal \& Altman, 2010; Catani, Jones, \& ffytche, 2005; Glasser \& Rilling, 2008; Thiebaut de Schotten, Dell'Acqua, Valabregue, \& Catani, 2012). However, in part because the methodology is relatively new, little is known about the development of these pathways, and their corresponding function in young children remains understudied.

\section{White Matter Development}

The organization and integrity of white matter tracts follows a protracted developmental timeline. It begins in the fetal period and continues into adulthood, but there are also regional differences in the timing of development (Kasprian et al., 2008; Lebel et al., 2012; Zhai, Lin, Wilber, Gerig, \& Gilmore, 2003). Research suggests that maturation of fiber tracts during the first 2 years of life in normal developing infants is characterized by three stages 1) rapid change during the first 12 months, 2) slow modifications during the second year, and 3) slower development after 24 months (Geng et al., 2012; Hermoye et al., 2006; Schneider et al., 2004). In addition to this characteristic temporal order, fiber tracts develop in region-specific ways (Dubois et al., 2008; Paus et al., 2001; Zhai et al., 2003). Flechsig (1896) showed that white matter development in infants begins first in "projection" pathways of the primary sensory and motor regions. Moreover, several "association" fiber pathways, which connect secondary 
regions of the cortex, are identifiable at birth. These include the superior longitudinal fasciculus, cingulum, corpus callosum, inferior fronto-occipital, and inferior frontal occipital fasciculus (Dubois et al., 2008; Geng et al., 2012; Hermoye et al., 2006). A number of these pathways show reduced white matter integrity at birth. For example, FA values are highest in deep white matter structures (e.g., corpus callosum) and lowest in frontal-temporal connections (e.g., the superior longitudinal fasciculus and inferior longitudinal fascicles; Dubois et al., 2008; Hermoye et al., 2006; Schneider et al., 2004). These pathways also show prolonged developmental trajectories, with frontal-temporal and frontal-parietal connections showing extended age-related change (Barnea-Goraly et al., 2005; Lebel et al., 2012).

After infancy, age-related changes in white matter development continue through childhood and into early adulthood (Nagy, Westerberg, \& Klingberg, 2004; Paus et al., 1999; Reiss, Abrams, Singer, Ross, \& Denckla, 1996), but again maturation is regionally specific, with some fiber pathways developing to maturity later than others (BarneaGoraly et al., 2005; McGraw, Liang, \& Provenzale, 2002; Wilke, Krägeloh-Mann, \& Holland, 2007). For example, of the association tracts, the inferior longitudinal and fronto-occipital fasciculi are the first to mature, while the superior longitudinal fasciculus/arcuate fasciculus (SLF/AF) and the uncinate fasciculi are the last (Lebel et al., 2012). Optimal white matter development has many advantages. For example, increases in myelination and axonal thickness concomitant with improved organization of white matter tracts result in increased efficiency and speed of neural transmission (Aboitiz, Scheibel, Fisher, \& Zaidel, 1992; Schmithorst, Wilke, Dardzinski, \& Holland, 2005). Secondly, several studies have related these changes to behavioral changes in children. 
For example, age-related changes in white matter integrity have been associated with visual information processing efficiency (Mabbott, Noseworthy, Bouffet, Laughlin, \& Rockel, 2006), working memory capacity (Klingberg, Vaidya, Gabrieli, Moseley, \& Hedehus, 1999; Nagy et al., 2004), and overall intelligence (IQ; Schmithorst et al., 2005). Researchers have also explored speech, language, and literacy correlates of white matter development. For example, age-related changes in white matter integrity have been associated with auditory processing ability (Schmithorst, Holland, \& Plante, 2011), phonological awareness skill (Yeatman et al., 2011), reading speed (Nagy et al., 2004), and accuracy reading words and pseudowords (Qiu, Tan, Zhou, \& Khong, 2008; Yeatman et al., 2011). However, maturation should not be considered as the sole contributor to enhanced performance. For example, several studies found that white matter integrity significantly predicted working memory capacity (Klingberg et al., 1999) or phonological awareness (Yeatman et al., 2011), even after controlling for age. Therefore, the evidence suggests that other factors aside from maturation (e.g., experience) account for observed brain/behavior relations.

While these studies have significantly contributed to our understanding of white matter development in relation to behavioral changes, they have typically been studied in older children. They have also failed to develop a comprehensive picture of how the known perisylvian language pathways develop and contribute to a broader language profile - that is, a profile that establishes the child's skill in multisensory speech processing, phonological and articulatory ability, receptive and expressive language ability, and literacy. Therefore, the present study aims to establish a better understanding of how perisylvian language pathways relate to speech, language, and literacy. In the next 
section we review which fiber pathways are thought to anchor the perisylvian language network in the human brain.

\section{Contemporary Model of the Neurobiology of Language}

Our investigation is framed within a contemporary model for the neurobiology of language, which is a dual stream model analogous to the model for visual processing (Dick \& Tremblay, 2012; Dick, Bernal, \& Tremblay, 2013; Hickok \& Poeppel, 2007; Poeppel, Emmorey, Hickok, \& Pylkkänen, 2012). The dual stream model examines dorsal and ventral processing streams. The dorsal stream involves interactions between frontal, parietal, and temporal brain regions and has been implicated in mapping auditory speech sounds to articulatory (motor) representations and also in processing complex syntactic structures. The most prominent pathway proposed to connect these regions is the superior longitudinal fasciculus/arcuate fasciculus (SLF/AF). In comparison, the ventral stream has been implicated in mapping auditory speech sounds to meaning and also in processing less complex syntactic structure. The most prominent pathways within the ventral stream are the uncinate fasciculus (UF), the extreme capsule (EmC), the middle longitudinal fasciculus (MdLF), the inferior longitudinal fasciculus (ILF), and the inferior fronto-occipital fasciculus (IFOF). A third set of fiber pathways comprising the motor stream establish the connections necessary for speech production, and include the cortico-bulbar system, the cortico-striatal loops, and the cortico-cerebellar system. A newly discovered association pathway of the cortex, the frontal aslant tract (FAT), has been proposed as an important pathway in the motor stream. See Figure 1 below. 


\section{Long-Association Fiber Pathways of the Perisylvian Cortex}

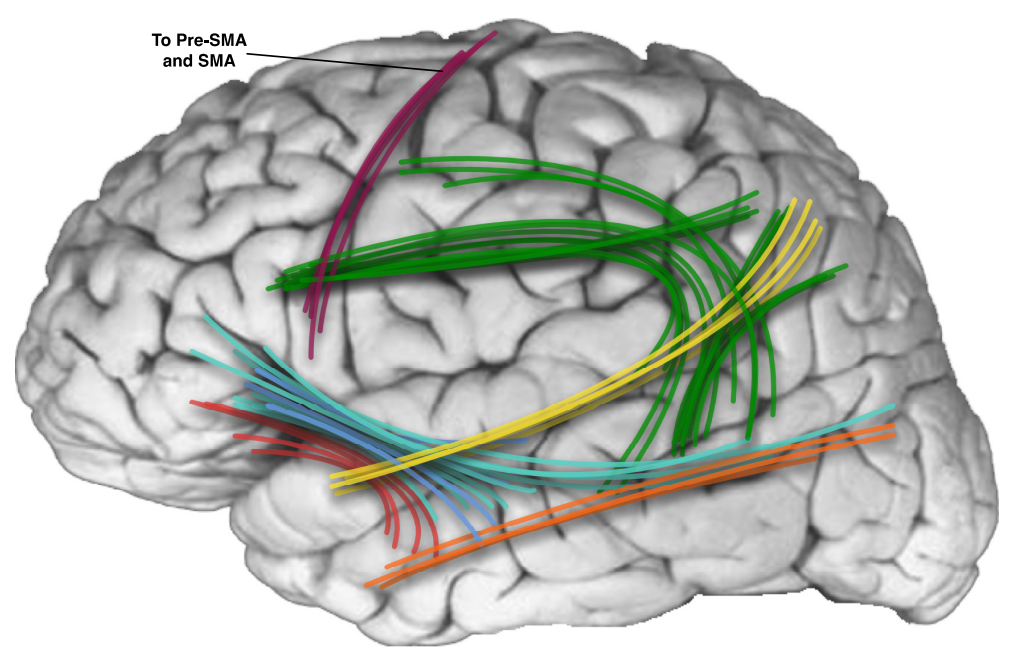

Superior longitudinal/arcuate fasciculus

Extreme capsule fiber system

Inferior longitudinal fasciculus

Inferior fronto-occipital fasciculus

Middle longitudinal fasciculus

Uncinate fasciculus

Frontal aslant tract

Figure 1. Lateral view of the left hemisphere showing the most prominent fiber pathways for language. The current study focuses on two: the SLF/AF in green and the FAT in purple. Reprinted from Dick and Small, in press.

\section{Fiber Pathways for Speech, Language, and Literacy}

From the set of fiber pathways that serve the processing of language, from early speech perception to semantic processing to narrative-level understanding, the present study focuses on two main pathways that are most associated with developing speech, language, and literacy abilities. The first is the well-known SLF/AF pathway that anchors the dorsal stream and is thought to be involved in phonological processing, receptive and expressive language processing, and literacy. The second pathway is the newly described Frontal Aslant Tract (FAT) of the motor speech stream (see Figures 2 and 3). Below we briefly review what is known about the putative functions and development of these two pathways with respect to speech, language, and literacy. 


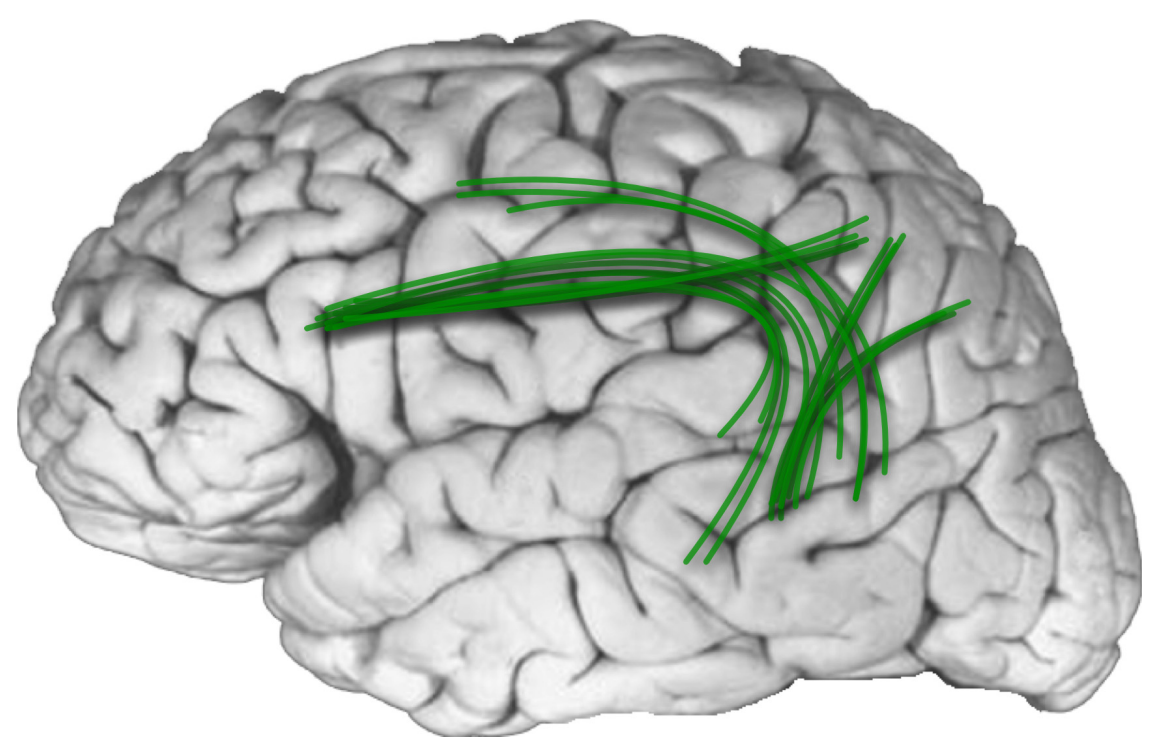

Figure 2. Connections of the SLF/AF in lateral view.

\section{Frontal Aslant Tract}
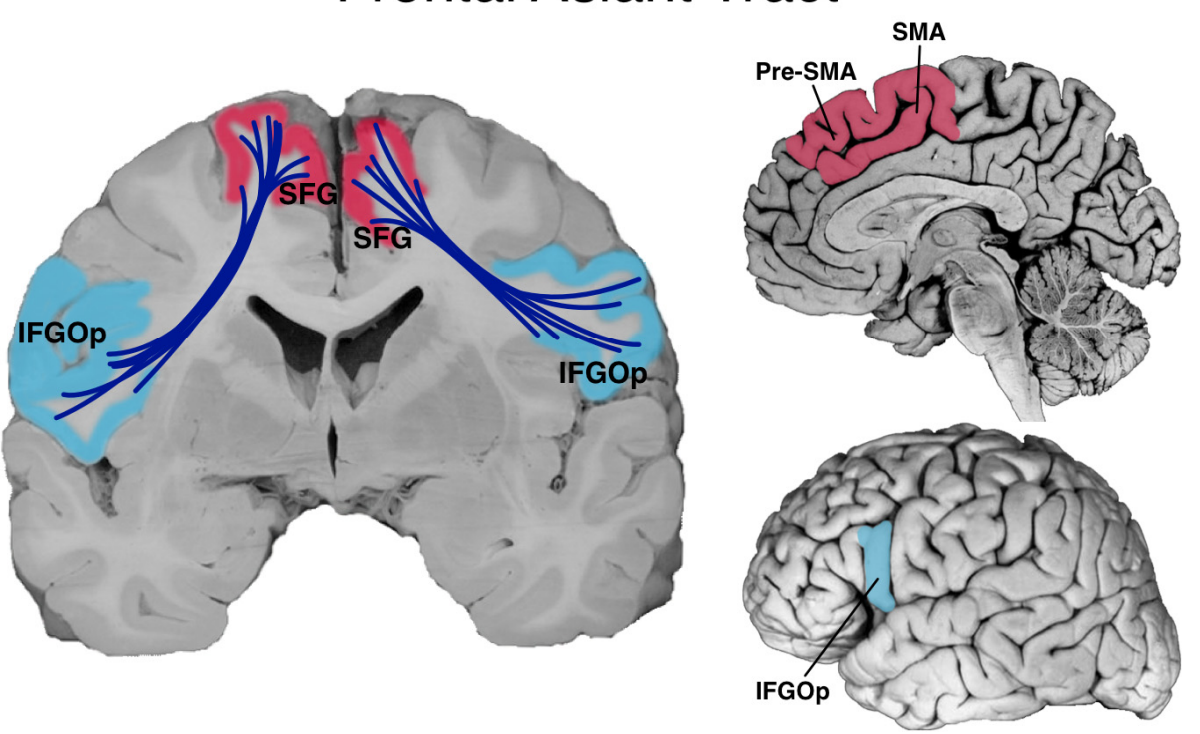

Figure 3. Connections of the frontal aslant tract (FAT) in coronal section, with outline of the inferior frontal and superior frontal origins and terminations in the medial and lateral sagittal views. IFGOp = inferior frontal gyrus, pars opercularis; $\mathrm{SFG}=$ superior frontal gyrus; SMA = supplementary motor area; Pre-SMA = pre-supplementary motor area. 


\section{Behavioral and Neural Components of Speech, Language, and Literacy}

Behavioral Components of Speech. It is often underappreciated that speech development occurs in a multisensory context. Infants develop in a multisensory environment, and early speech perception experiences are often accompanied by visual speech information. A very large corpus of research suggests that multisensory stimulation provides tangible benefits to the developing child (Bahrick, Lickliter, \& Flom, 2004; Blomert \& Froyen, 2010; Erdener \& Burnham, 2013; Gogate \& Bahrick, 1998; Gogate, Bahrick, \& Watson, 2000; Lachs, Pisoni, \& Iler Kirk, 2001; Magnan \& Ecalle, 2006; Magnan, Ecalle, Veuillet, \& Collet, 2004; Nath, Fava, \& Beauchamp, 2011; Ross et al., 2011; Sekiyama \& Burnham, 2004). For example, amodal information, which refers to information shared across multiple sense modalities (i.e., temporal synchrony, rhythm, tempo), seems to have the effect of directing attention to what is important in the environment, and enhancing salient features of actions and objects (Bahrick et al., 2004; Calvert, Campbell, \& Brammer, 2000). Broadly, the detection of temporal synchrony seems to be an important skill in producing meaningful, unified percepts (Bahrick \& Lickliter, 2004). For example, during synchronous AV speech stimulation, visual input from a speaker's mouth and lips has the effect of speeding up and enhancing auditory neural processing, indexed by shorter latencies and larger amplitudes compared to unisensory auditory stimulation (van Wassenhove, Grant, \& Poeppel, 2005). These results in turn may have the affect of facilitating categorical learning of speech information.

Early detection and identification of synchronous AV speech information seems to facilitate the perception and learning of more complex speech skills. The impact of 
visual speech influence on speech perception seems to emerge early, around 4-months of age (Bahrick, 2010; Kuhl \& Meltzoff, 1982). By 4-months, infants successfully match congruent speech information (i.e., visual /a/, auditory /a/) versus incongruent (i.e., visual /i/, auditory /a/) speech information (Kuhl \& Meltzoff, 1982). In the same study, Kuhl and Meltzoff (1982) also found that infants spontaneously produced sounds that resembled those of the female actress articulating the vowels, with respect to intonation, contours, and overall durations. These findings suggest that infants may be making use of the visual articulatory information from the speaker's lips and mouth and auditory information from the sound of the speaker's voice to produce the sounds themselves. Findings from Kuhl and Meltzoff (1982) are consistent with the intersensory redundancy hypothesis, which suggests that the overlap of amodal properties (e.g., temporal synchrony, rhythm, or intensity) guides what we attend to, perceive, learn and remember (Bahrick 2010). Therefore, congruent and synchronous AV speech (e.g., congruent audiovisual/ba/) may help recruit attention (Bahrick 2010), speed up neural processing (van Wassenhove, Grant, \& Poeppel, 2005), and help with learning properties across a third domain (e.g., articulatory /ba/). These data suggest that early sensitivity to multisensory AV speech information, such as the detection of synchrony and other linguistic properties of speech, may serve as a strong foundation, a bootstrapping effect, for later, more sophisticated speech and literacy skill in childhood and adulthood.

The influence of multisensory audiovisual speech on auditory speech perception continues to show age-related change well into middle childhood and adulthood, but there seems to be rapid development in the period between 5- and 8-years. For example, there are age-related differences in the degree to which visual speech information 
enhances the perception of auditory speech, such that children benefit significantly less from observing visual information than adults (Desjardins, Rogers, \& Werker, 1997; Massaro, Thompson, Barron, \& Laren, 1986; McGurk \& MacDonald, 1976; Sekiyama \& Burnham, 2008). In fact, younger children (5-years-old) seem to take less advantage of visual speech information than older children (8-years-old) (Van Linden \& Vroomen, 2008). It has been suggested that preschool children (age 5) have less experience producing speech compared to older children, and as a result do not benefit from AV speech to the same degree (Desjardins et al., 1997; Van Linden \& Vroomen, 2008). Therefore, the degree of experience with multimodal speech matters, and the research suggests that the multimodal speech perception system is one that takes years to develop into full maturity, but that also shows significant development between 5- and 8-years.

Neural Components of Speech. Several brain regions have been implicated in AV speech, predominately the inferior frontal/ventral premotor, inferior parietal, and posterior temporal regions, and these regions have been proposed to be connected by the SLF/AF (Bernal \& Altman, 2010; Catani et al., 2005; Dick \& Tremblay, 2012; Martino et al., 2013; Thiebaut de Schotten et al., 2012). Two processing streams, namely the auditory-visual and auditory-motor pathways, ground functional interactions among this frontal-parietal-temporal network. The first, the sensory-sensory (auditory-visual) pathway is anchored by the posterior superior temporal sulcus (STSp), and connects visual domains (especially MT/V5), primary auditory cortex, STSp, and inferior frontal regions (Arnal, Morillon, Kell, \& Giraud, 2009; Calvert, 1997). Evidence in older children (Nath \& Beauchamp, 2012; Nath et al., 2011) and adults (Arnal et al., 2009; Barrós-Loscertales et al., 2013; Calvert, 1997) suggest that auditory and visual 
information are integrated into perceptual units in the STSp. For example, the amplification of the activation in STSp has been associated with perceiving McGurk fusions. McGurk fusions occur when perceivers are presented with incongruent visual and auditory stimuli (e.g., visual/da/, auditory /ba/), and as a consequence report hearing a syllable that is different from the original stimuli (e.g., ga).

The second, the "sensory-motor" stream has been suggested to be involved in mapping the perceptual units of sound (i.e., phonological information) in the auditory modality to articulatory representations involved in producing those sounds (Callan, Jones, Callan, \& Akahane-Yamada, 2004; Skipper, van Wassenhove, Nusbaum, \& Small, 2007). This stream is anchored by the inferior supramarginal gyrus/planum temporale (iSMG/PTe), and connects inferior frontal gyrus, premotor cortex, anterior insula, and in some reports precentral and somatosensory brain regions to the posterior planum temporal (Spt), and focal regions of the STG and STS (Buchsbaum, Hickok, \& Humphries, 2001; Callan et al., 2004; Dronkers, 1996; Hickok \& Poeppel, 2000; Wilson, 2004).

The auditory-motor network has been primarily implicated in planning and executing speech movements (e,g., speech production). However, several lines of neuroscience research suggest that motor regions play a crucial role in AV speech perception as well, namely through functional interactions between auditory, motor, and somatosensory brain regions (Callan et al., 2004; Skipper, Nusbaum, \& Small, 2005; Skipper et al., 2007; Watkins, Strafella, \& Paus, 2003) and with the iSMG/PTe as an important structure facilitating integration (Griffiths \& Warren, 2002; Karbe, Herholz, Weber-Luxenburger, Ghaemi, \& Heiss, 1998; Okada \& Hickok, 2009). The FAT tract in 
particular may contribute to audiovisual processing by facilitating interactions between speech-motor regions of the supplementary motor areas and the inferior frontal gyrus. However, to date this has not been investigated.

In summary, two processing streams seem to be involved in auditory and audiovisual speech perception, namely the auditory-visual and auditory-motor pathways and these pathways are connected by the SLF/AF. While the SLF/AF has been proposed to connect the described speech network, we do not know if there are structural differences in the SLF/AF related to speech in the developing child's brain, and in particular there is no evidence linking the development of these fiber pathways to agerelated changes in audiovisual speech perception. It is also notable that no studies have identified the FAT in children of any age, nor attempted to relate the integrity of this tract to developing speech. Given its putative involvement in speech production, the FAT may also contribute to speech perception, especially in children who are continuing to develop articulatory and phonological skill.

Behavioral Components of Language. Language is a complex cognitive task that requires processing multiple levels of information, such as word-level processing, sentence processing, and discourse processing. Development of language proceeds across these levels. Thus, infants can learn to discriminate speech sounds by 4-6 months, and by 6-8 months, infants begin to babble, or produce sounds that are within their native inventory of phonemes (Bates, Benigni, Bretherton, Camaioni, \& Volterra, 1979; Lewedag, Oller, \& Lynch, 1994; Masataka, 2001). Comprehension at the word level emerges around 8-10 months (Bates, Benigni, Bretherton, Camaioni, \& Volterra, 1979), and production of words occurs a few months later at 11-13 months (Bates \& Dick, 2002; 
Shore, Bates, Bretherton, Beeghly, \& OConnell, 1990). With age, more complex skills emerge. For example, at 18-20 months, infants begin to combine words together (Bauer \& Thal, 1990), and by 24 months, the combinations of words follow the appropriate grammatical structure of their native language (Bauer, Wenner, Dropik, \& Wewerka, 2000). While the developing child begins to produce meaningful and grammatically correct utterances by 24 months, we must acknowledge that there are individual differences in toddlers' receptive and expressive language, meaning their ability to understand and produce language, and this is predictive of children's later receptive and expressive language in early school years.

Neural Components of Language. The neural network for expressive and receptive language includes the fronto-temporo-parital neural network reviewed for speech perception and production, including the inferior frontal/ventral premotor, inferior parietal, and posterior temporal regions. However, processing beyond phonological-level information, at the semantic and syntactic level, includes more extensive perisylvian language cortical regions including the occipito-temporal and middle and inferior temporal regions (Catani \& Mesulam, 2008; Dick, Solodkin, \& Small, 2010; Glasser \& Rilling, 2008; Hickok \& Poeppel, 2004). Some additional projections to the SLF/AF have been associated with higher-level language processing. For example, neuroimaging evidence in addition to evidence from adults with stroke has found that damage to the SLF is related to deficits in repetition of speech, while the AF components seems to be important for comprehension of speech (Breier, Hasan, Zhang, Men, \& Papanicolaou, 2008). Only one study has related the FAT to behavioral measures of language (Catani et al., 2013). Catani and colleagues characterized the FAT in adults with Primary 
Progressive Aphasia and found that white matter integrity of the FAT was associated with the expressive language measure of verbal fluency (Catani et al., 2013). As already mentioned, no study of the FAT has been conducted in children, but given the association with expressive language in adults with aphasia, we might expect the FAT to support developing expressive language in children.

Behavioral Components of Literacy. Literacy is developing at the same time as language and the ability to read requires children to develop several skills. First, the child must know the phonological structure (i.e., the units of sounds) of a given language regardless of language dominance. In alphabetic languages, such as English or Spanish, children must also learn to map letters to speech sounds (i.e. phonemes), and then assemble these sounds serially, letter-by-letter to create meaningful words (Durgunouglu, Nagy, \& Hancin-Bhatt, 1993; Liberman \& Shankweiler, 1987; Read, Yun-Fei, Hong-Yin, \& Bao-Qing, 1986). The ability to recognize and manipulate the sound structure of spoken words is called phonological awareness (Mattingly, 1972), and has been found to be the most significant predictor in later reading and vocabulary skill in school-aged kindergarten children (Lundberg, Olofsson, \& Wall, 1980). In fact, these effects are also detected over an extended developmental timeline; so much so that early phonological awareness predicts literacy outcomes from kindergarten through the fourth grade and remain remarkably stable (Wagner et al., 1997). Therefore, children, especially young children, may profit from phonological training even before beginning to read, and further may profit from a continuation of phonologically mediated training through the elementary school grades. These results have important implications not only for preschool instruction, but also for developing literacy and teaching programs geared 
toward adults and individuals with reading disabilities. Importantly a number of gains in literacy are occurring in the 5-8-year age range we target in the present study.

Neural Components of Literacy. A growing body of literature has investigated the structural connectivity supporting literacy in children and found that fibers traveling anterior to posterior in the temporal and parietal cortex are associated with reading (Klingberg et al., 2000). These fibers, which travel as part of the SLF/AF, have been proposed to connect inferior frontal/ventral premotor, supramarginal gyrus and angular gyrus in the parietal lobe, occipital temporal areas, and posterior temporal cortical regions (Shaywitz \& Shaywitz, 2008), and they play a critical role in phonological awareness (e.g., rhyming tasks) and reading (Beaulieu et al., 2005; Shalom \& Poeppel, 2008; Temple et al., 2003).

White matter integrity of the SLF/AF in the temporal-parietal region of the left hemisphere has been significantly related with reading scores in reading-impaired children and adults and typically developing children (Klingberg et al., 2000; Nagy et al., 2004; Thiebaut de Schotten, Cohen, Amemiya, Braga, \& Dehaene, 2012). One case study reported that a 5-year-old acquired severe dyslexia after damage to the arcuate fasciculus (Rauschecker et al., 2009). Taken together, the aforementioned evidence suggests that the inferior frontal-parietal-temporal network, connected by the perisylvian SLF/AF is important for reading. In contrast, the FAT has not been associated with literacy, and there is little reason to suspect that it might be, as it does not provide connectivity to temporal and parietal regions most important for reading. 


\section{CHAPTER III}

\section{HYPOTHESES AND PREDICTIONS}

As our review shows, there is a growing literature investigating developing fiber pathways and the relation of these to speech, language, and literacy. However, the vast majority of these studies have investigated older children (e.g., older than 8 years). This is often done for practical reasons, as it is difficult to scan younger children because of issues with MRI image artifacts as a result of movement during the scan. As our literature review suggests, though, this focus on older children misses the considerable development in speech, language, and literacy occurring in younger age ranges, particularly from 5-8-years. Although these behavioral-level trends are well established, almost no work has investigated the developing fiber pathways that contribute to this development.

To fill the considerable knowledge gap, the present study aims to characterize the specific age-related changes in SLF/AF and the FAT white matter microstructure and relate white matter microstructure of both pathways to speech, language, and literacy abilities. The most prominent fiber pathway identified with language and literacy is the $\mathrm{SLF} / \mathrm{AF}$. The SLF/AF has been investigated in children, but these children are much older than we investigate here (typically 8-11-years old), and there is often little investigation of the relation to specific behavioral measures of language, or if behavioral measures are used, they are gross language measures (e.g., verbal IQ). The FAT is newly described in adults, but has never been shown in children. Therefore, the aims of the present study are to: 1) identify the FAT in young children who are still developing speech, language, and literacy, 2) relate the FAT to speech, language, and literacy, 3) 
characterize the SLF/AF, and 4) relate the SLF/AF to speech, language, and literacy. The measures of speech, language and literacy skill that we investigate include performance on AV speech perception task, and performance on a battery of tests assessing phonology, articulation, receptive and expressive language, and phonological awareness. We predict that, after controlling for age, whole brain white matter integrity, nonverbal IQ, and sex, higher white matter integrity (higher FA values; lower ADC values) of the SLF/AF and FAT will be related to better speech, language and literacy skills. The exception to this is that we did not expect a relation between FAT white matter integrity and literacy. The latter hypothesis is supported by our review of the neural components of literacy, which predominately include connections among inferior frontal/ventral premotor, supramarginal gyrus and angular gyrus in the parietal lobe, occipital temporal areas, and posterior temporal cortical regions (Shaywitz \& Shaywitz, 2008). While the FAT has been proposed to connect supplementary motor and pre-supplemetary motor areas with the inferior frontal gyrus, it does not make connections to inferior parietal and occipitotemporal regions involved in literacy. Therefore, we do not expect a relation between the FAT white matter integrity and literacy. 


\section{CHAPTER IV}

\section{METHOD}

\section{Participants}

Nineteen children ( 9 females, 10 males; age range $=5-8$ years, $M$ age $=6.8$ years, $\mathrm{SD}=1.1$ years) comprised the final sample. All participants were screened by phone for contraindication to MRI, were right-handed according to the Edinburgh Handedness Inventory, bilingual English/Spanish speakers with normal hearing (self-reported), and had normal (or corrected to normal) vision. An additional 3 children completed the diffusion weighted scan but were not analyzed because of image artifacts indicated after the scan (one was due to an error of the technician; two were removed due to motion artifact). An additional 11 children were consented but did not complete the diffusion weighted scan because of their refusal to assent, or because of significant movement during the T1-weighted structural scan before the diffusion-weighted scan was initiated. Written informed consent/assent was obtained from all parents and children for whom we have usable data. The Western Institutional Review Board and the Florida International University Institutional Review Board approved the study.

\section{Procedure}

Data were collected during two visits. The first visit took place at Miami Children's Hospital, Miami, FL and consisted of three sessions: 1) a pre-test session audiovisual speech categorization task ( $\sim 8 \mathrm{~min})$ to determine individual's categorical speech perception, 2$)$ a scanning session ( $\sim 25 \mathrm{~min})$, and 3$)$ a post-test session ( $\sim 4 \mathrm{~min})$ to determine visual influence of speech. The second visit was scheduled within two weeks of the first visit and took place at Florida International University. During this visit ( 90 
min), we administered a battery of language tests measuring phonology, articulation, receptive and expressive language, and reading abilities.

Stimuli for the Audiovisual Speech Task. Six adults (3 females) were recorded speaking syllables (/ba/, /da/, /pa/, /ka/) with a digital video camera under two conditions (Visual Speech [VS] and Masked Speech [MS]). In the MS condition, speakers wore a surgical mask to hide lip and mouth speech-related movements. In the VS condition, speakers did not wear a surgical mask. Two additional speakers ( 1 female) were recorded saying these same syllables with a remote microphone. The consonant-vowel (/ba/,/da/, $/ \mathrm{pa} /, / \mathrm{ka} /$ ) utterances were selected because the place of articulation of the consonant varied along a single factor. The same vowels were selected because the formant structure has been shown to provide superior signal/noise ratio relative to the MRI scanner during functional runs (Miller \& D'Esposito, 2005).

From these audio recordings, two naturalistic 7-step continua (ba $\longleftrightarrow$ da; pa $\longleftrightarrow$ ka) were developed using Praat software. The start of each vowel /a/ was clipped to isolate the consonant. Then the vowel from one recording was affixed to each consonant. Affixing the vowel from one recording to each consonant ensured that identical vowels were heard across all constants. Then we used "Pitch Synchronous Overlap and Add" to modify the pitch and duration of each sound, and interpolated between these two modified sounds to develop the continua. By combining the audio clips from the continua with the video clips from the six speakers, we created the audiovisual clips. For both VS and MS conditions, the consonantal burst of the auditory file was aligned to the consonantal burst of auditory portion of the video file. All clips were edited to last 1.6 seconds. 
Pre-and-post-test categorical syllable perception. The categorical syllable perception task included pre-and post-test sessions. The purpose of the pre-test was to identify each individual's speech categorical boundary. To do this, we used the MS stimuli to create a second factor, Ambiguity. We used the MS to avoid visual influences from the lips and mouth, and therefore better capture their auditory syllable perception. Along the seven step pa $\longleftrightarrow \mathrm{ka}$ or $/ \mathrm{ba} / \longleftrightarrow \longrightarrow$ da speech continua, the first and last steps were designed as unambiguous. Steps in between the first and last were considered ambiguous because as you move along the pa $\longleftrightarrow$ ka continuum, the /pa/ starts to sound more like a $/ \mathrm{ka} /$. However, while some participants may hear the $/ \mathrm{pa} /$ sound change to a $/ \mathrm{ka} /$ sound at the third step of the continuum, others might hear it change at the fourth and fifth step. The two ambiguous sounds along the continuum in which the participant shifts from one category (e.g., /pa/) to a different category (e.g., /ka/) defined their speech categorical boundary. Therefore, to determine the pre-test categorical boundary, participants were presented with MS stimuli. Each step along the continuum was presented 3 times in a pseudo-randomized sequence for a total of 48 trials across the two continua ( $\sim 8$ mins). Children were asked to watch the video clip and repeat out loud the sound they heard. Their responses determined which step along the 7 step continua (e.g., $/ \mathrm{ba} / \longleftrightarrow \longrightarrow / \mathrm{da} /$ ), the child shifted from one speech category (e.g., /ba/) to a different speech category (e.g., /da/), without the influence of speech-related lip and mouth movements. The two ambiguous sounds at the category boundary along with two unambiguous sounds (the ends of the continua) were presented to each participant during the post-test session. 
The purpose of the post-test session was to calculate the degree to which visual information from the lips and mouth influenced participant's categorical speech perception. For the post-test, children were presented with the VS and MS stimuli using only the ambiguous and endpoints of the continuum (48 stimuli total, $\sim 8 \mathrm{mins}$ ).

Therefore, each participant heard different sounds depending on how they categorized them in the pre-test. Once more, children were asked to watch the video clip and repeat out loud the sound they heard. For each stimulus, the difference in steps along the continuum across the VS and MS condition serves as a measure of AV integration.

Assessments. The following assessments were administered to obtain a complete understanding of each child's speech and language ability: the Diagnostic Evaluation of Articulation and Phonology (DEAP; Dodd, Zhu, Crosbie, Holm, \& Ozanne, 2002), including articulation, and phonology subtests, the Clinical Evaluation of Language Fundamentals-4 (CELF-4; Semel, Wiig, \& Secord, 2003), including receptive language (REC), expressive vocabulary (EXP), concept and directions, word class, and sentence structure subtests, the Wechsler Preschool and Primary Scale of Intelligence Third Edition (WPPSI - III; Wechsler, 2002) Block Design subtest, and the Word Attack (Test 3), Sound Awareness (Test 7), and Sound Blending (Test 8) subtests of the WoodcockJohnson III Diagnostic Reading Battery (WJ III DRB; Schrank \& Mather, 2006). For this analysis, we used the DEAP for measuring phonology and articulation, the CELF for measuring receptive and expressive language, and the WJ-III for measuring literacy. We also used the block design subtest of the WPPSI-III to control for non-verbal IQ. Standardized scores were used in the analysis for all subtests. 
Data Acquisition. Participants were scanned on a 3 Tesla Philips MRI scanner with SENSE coil housed at Miami Children's Hospital. Prior to the actual scanning session, participants underwent a simulated scan in a mock scanner to familiarize them to the MRI scanner environment. In addition, vitamin E capsules were placed on participants' fronto-temporal left forehead to verify orientation of images during postprocessing.

Diffusion-weighted images were collected for detecting individual differences in white matter architecture. Images were acquired using single-shot spin-echo echo-planar imaging sequence $(15$ gradient directions, $b$ value $=900 \mathrm{~s} / \mathrm{mm} 2$ and $b=0 \mathrm{~s} / \mathrm{mm} 2$ (reference scan), matrix size $=112 \times 112$, time echo $[\mathrm{TE}]=60$, time repetition $[\mathrm{TR}]=$ $6157, \mathrm{NEX}=3, \mathrm{FOV}=240 \times 240 \mathrm{~mm} 2$, slice thickness $=2 \mathrm{~mm}$, number of axial slices $=$ 55 (no gap), and voxel size $=0.938 \mathrm{~mm} \times 0.938 \mathrm{~mm} \times 2 \mathrm{~mm}$ ). We also collected highresolution T1-weighted anatomical images for each participant using an 8-min sagittal 3D spoiled gradient recall (SPGR) sequence $(120$ axial slices, voxel size $=1.5 \times .938 \times$ $.938 \mathrm{~mm}$ resolution). Head motion was minimized by placing cushions around the head and securing a strap across the forehead. The duration of scanning time was approximately 25 min per subject, not including setup time.

Diffusion Tensor Imaging Post-Processing. We used FSL (http://fsl.fmrib.ox.ac.uk/fsl/fslwiki/), DSI Studio (http://dsi-studio.labsolver.org/), and Matlab (http://www.mathworks.com) software packages for all analyses. Diffusionweighted images were denoised using the Non-Local Means Filter adapted to Rician noise distribution (NLMr; Coupé, Manjón, Robles, \& Collins, 2011). The filter restores the intensity of voxels by computing a weighted average of all voxel intensities in the 
image, producing better quality fractional anisotropy maps (Descoteaux, Wiest-Daesslé, Prima, Barillot, \& Deriche, 2008; Wiest-Daesslé, Prima, Coupé, Morrissey, \& Barillot, 2008). Data were preprocessed also for eddy currents and subject motion, using affine registration to a non-weighted diffusion reference image $(b=0)$. Non-brain tissue was removed using FSL's automated brain extraction tool (BET). Using DSI Studio, we determined the tensors in each voxel using an over-determined linear equation system with least squares fitting (Jiang, van Zijl, Kim, Pearlson, \& Mori, 2006). The gradient table, which is necessary to extract the diffusion tensor, was computed using an open source PARtoNRRD toolbox in Matlab (Farrell et al., 2007). The diffusion tensor was used to calculate the eigenvalues reflecting diffusion parallel and perpendicular to each of the fibers along 3 axes $(x, y, z)$. The resulting eigenvalues were then be used to compute FA and ADC values (Basser et al., 1994).

We verified that the orientation and directions of tensors were correct using the fractional anisotropy red-green-blue (RGB) color map across all axes. We used welldefined fiber pathways for this verification, and inspected whether the cingulum was oriented along the anterior-posterior axis (labeled green) in the sagittal plane, whether the cortico-spinal tract (CST) was oriented along the superior-posterior axis (blue) in the coronal plane, and finally whether the corpus callosum was oriented along the mediallateral axis (red) in the axial plane.

Fiber Tract Identification. All fiber tracking procedures were performed in DSI Studio with a fractional anisotropy threshold of 0.15 and fiber angles of less than $40^{\circ}$ between connecting pixels. These thresholds are standard thresholds used by a prominent published atlas of white matter tracts (Oishi et al., 2008). 
Manual Fiber Tract Identification. We manually identified the SLF/AF for each individual using a one region of interest (ROI) approach. The SLF/AF region of interest was guided by fiber orientation maps and defined on the fractional anisotropy map computed from the averaged DT-MRI volume: red for medial-lateral, green for anterior-posterior, and blue for superior-inferior. We manually defined an ROI that encompassed all green voxels lateral to the internal capsule (Catani et al., 2005). The SLF/AF ROI was defined liberally, making sure to include all possible SLF/AF voxels and allowing voxels from neighboring tracts as well.

We also manually identified the FAT. We used a two-ROI approach (Catani et al., 2012; Thiebaut de Schotten et al., 2012). The first ROI was drawn in the posterior part of Broca's territory (i.e., precentral cortex, BA 6, pars opercularis, BA 44). The second ROI was drawn in the SMA and pre-SMA in the superior frontal gyrus (BA 8 and 6; Lawes et al., 2008; Oishi et al., 2008). All fibers passing through these ROIs were reconstructed in three dimensions and visualized using streamtubes. The streamtube method is used because it represents structures with primarily linear diffusion, that is the diffusion tensor along one direction in a 3D field (one eigenvalue) is much larger than the other two and therefore corresponds to linear anisotropy diffusion (Zhang, Demiralp, \& Laidlaw, 2003). White-matter tracts tend to produce tensors with linear anisotropy (Coremans, Luypaert, Verhelle, Stadnik, \& Osteaux, 1994).

\section{Data Analysis}

Use of Robust Statistics. We related our calculations of FA and ADC measures of white matter integrity to age and behavioral outcome measures using robust estimates of correlation $\left(r_{r o b}\right)$ and robust regression (using the Huber loss function). We used robust 
procedures because they are less influenced by outliers and increase the precision of parameter estimates when there are outliers (Wilcox, 2012; Wright \& London, 2009). We also calculated $95 \%$ confidence intervals using bootstrap methods for estimating the parameters (Efron, 1992; Efron \& Tibshirani, 1986). We used the R statistical package (v. 2.15.1; http://www.R-project.org) to conduct these analyses. 


\section{CHAPTER V}

\section{RESULTS}

The goal of the analysis was two-fold. First, we identified and characterized agerelated changes of white matter integrity in two fiber tracts important for language, the frontal aslant tract (FAT) and the superior longitudinal fasciculus/arcuate fasciculus (SLF/AF). Second, we related these changes to developing speech, language, and literacy.

\section{Identification of the FAT and SLF/AF Tracts}

We were able to track both the left $(\mathrm{M} \mathrm{FA}=0.40, \mathrm{SD}=.02 ; \mathrm{M} \mathrm{ADC}=0.84+/-$ $\left.0.02 \times 10^{-3} \mathrm{~mm}^{2} / \mathrm{s}\right)$ and right $(\mathrm{M} \mathrm{FA}$ values $=0.40, \mathrm{SD}=.02 ; \mathrm{M} \mathrm{ADC}=0.84+/-0.02 \times$ $10^{-3} \mathrm{~mm}^{2} / \mathrm{s}$ ) FAT in 17 out of 19 children. See Figure 4 on the next page. In one child we were not able to track the left FAT, but we were able to track the right FAT; in a different child we were not able to track the right FAT, but we were able to track the left FAT. However, it is notable that both of these FAT tracts appeared when the step size was adjusted to a more liberal level (of $2 \mathrm{~mm}$ ). See Figure 5 on the next page. Thus, the lack of identification should not be taken to indicate an absence of the tract, but may indicate an artifact in the data, noise in the data, reduced myelination of the tract, or Type II statistical error.

We were also able to reliably track the left $(\mathrm{MFA}=0.45, \mathrm{SD}=.02 ; \mathrm{M} \mathrm{ADC}=$ $\left.0.82+/-0.02 \times 10^{-3} \mathrm{~mm}^{2} / \mathrm{s}\right)$ and right $(\mathrm{M} \mathrm{FA}=0.44, \mathrm{SD}=.02 ; \mathrm{M} \mathrm{ADC}=0.82+/-0.02 \mathrm{x}$ $10^{-3} \mathrm{~mm}^{2} / \mathrm{s}$ ) SLF/AF in all children (see Figure 6). Both FA and ADC values for this tract fell within the normal range for children (Morriss et al., 1999; Sener, 2001). 


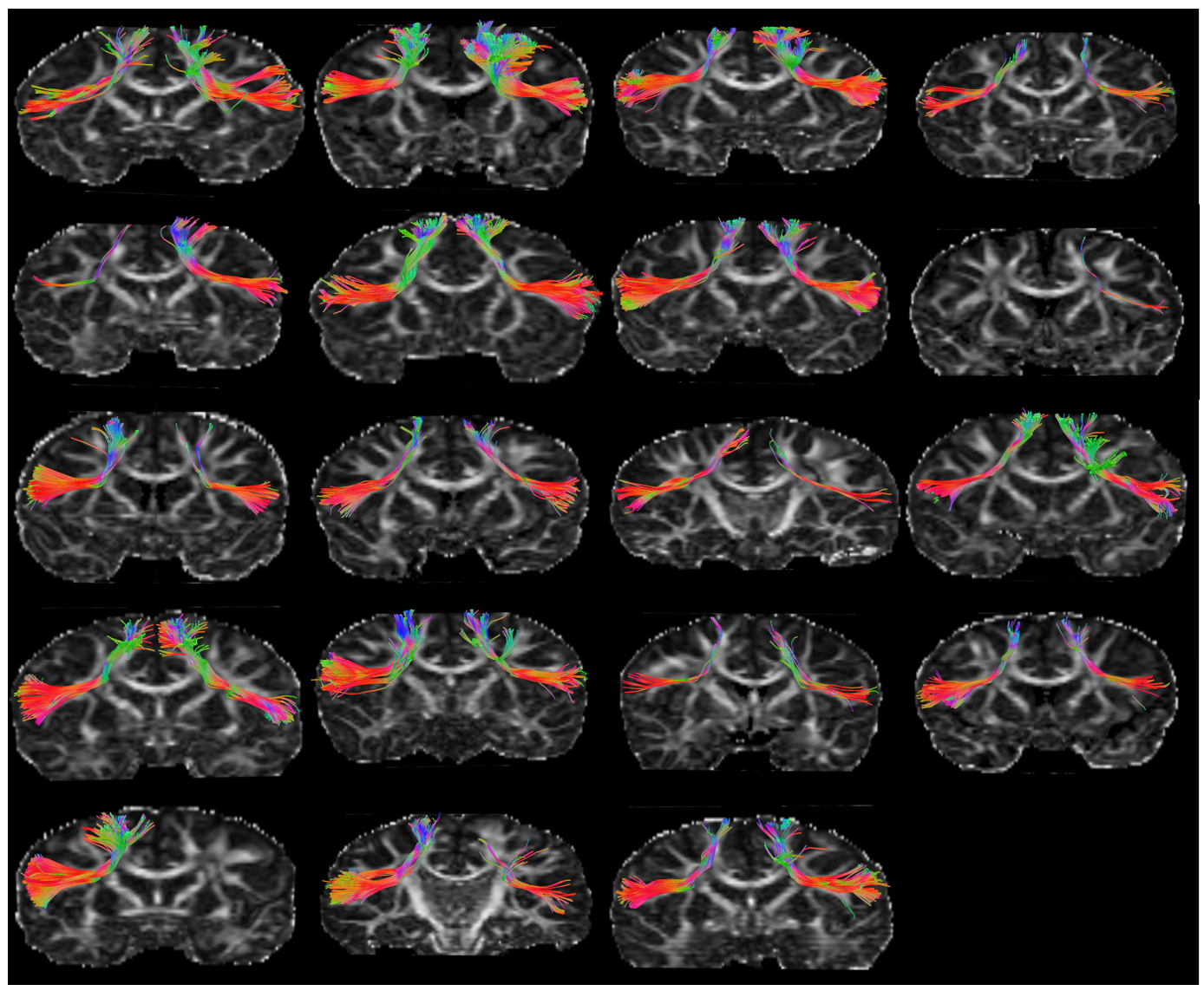

Figure 4. Connections of the Frontal Aslant Tract (FAT) in 19 children. Note: This

mosaic is displayed in radiological orientation. Therefore, the right side of the image represents the left cerebral hemisphere.

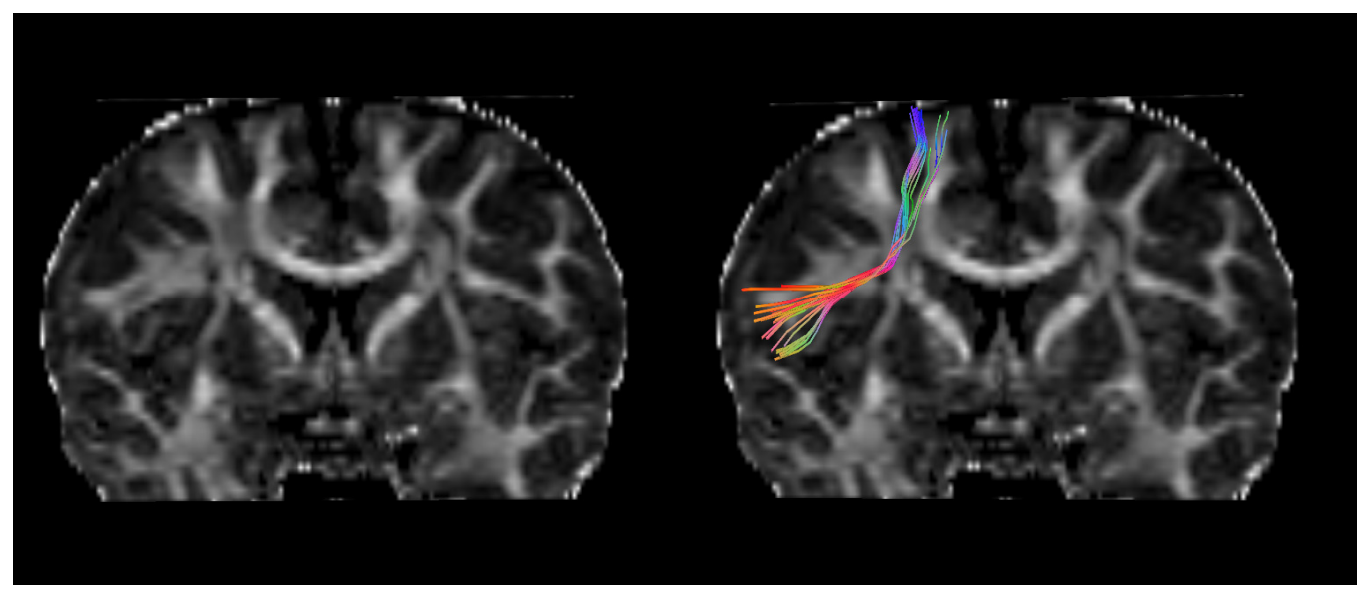


Figure 5. Left: FAT of one child was not identified in the right hemisphere under more stringent step size (of $1 \mathrm{~mm}$ ). Right: FAT appeared when the step size was adjusted to a more liberal level (of $2 \mathrm{~mm}$ ). Note: Figures are displayed in radiological orientation.

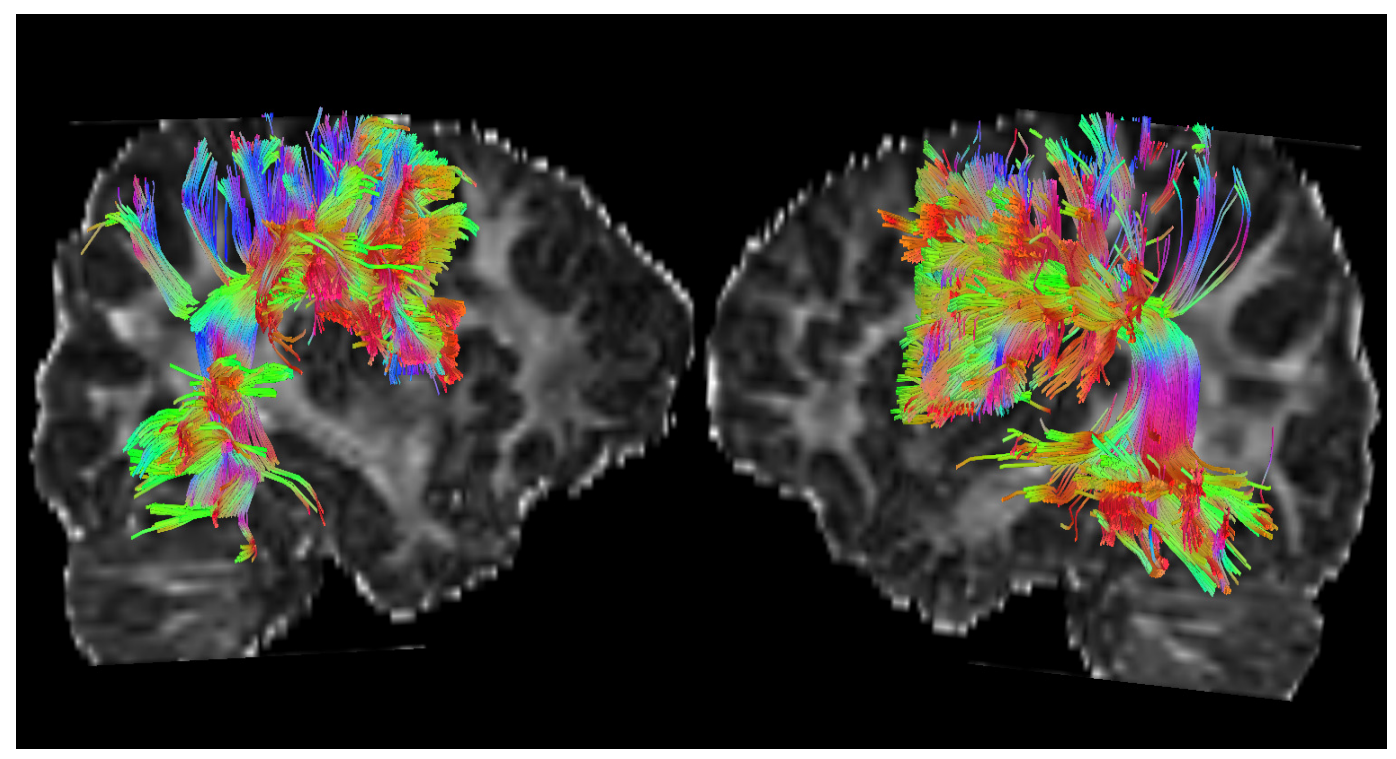

Figure 6. Left and right connections of the SLF/AF in one child.

\section{Age-related Change in the FAT and SLF/AF}

To characterize age-related changes of the FAT and SLF/AF we ran robust correlations. Figures 7 and 8 show robust elliptical plots (relplots; (Goldberg \& Iglewicz, 1992). Neither the left nor the right FAT showed age-related changes in white matter integrity, measured by FA values (Figure 7) and by ADC values (Figure 8). In contrast, the left SLF/AF did show age-related changes in white matter integrity, measured by FA values $\left(r_{r o b}=.88 ; \mathrm{p}<.001\right)$, but the right SLF/AF did not (Figure 7). Neither tract showed significant age-related change in ADC (Figure 8). 

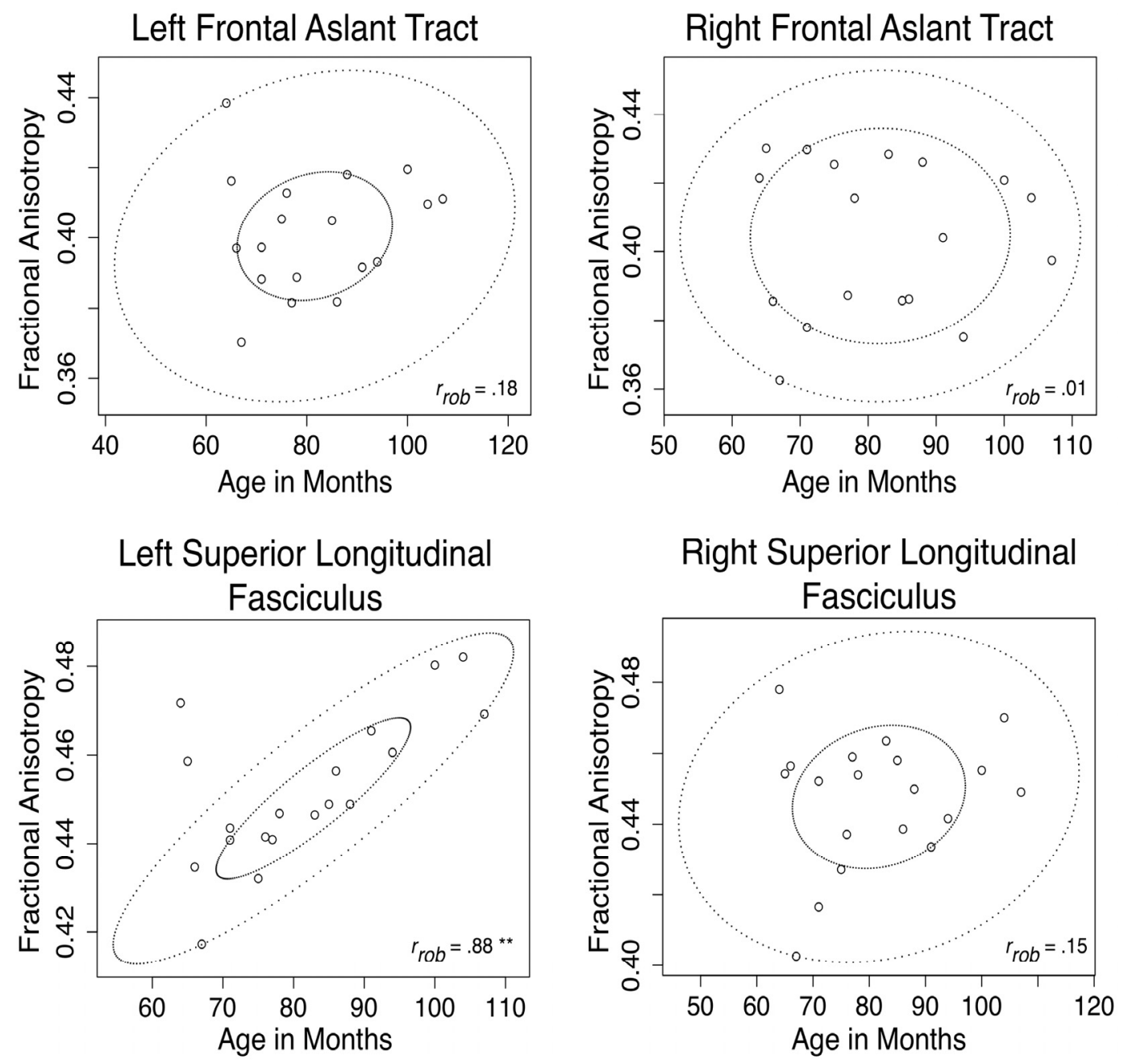

Figure 7. Top: Age-related changes in fractional anisotropy in the left and right Frontal Aslant Tract. Bottom: Age-related changes in fractional anisotropy in the left and right Superior Longitudinal Fasciculus/Arcuate Fasciculus. 

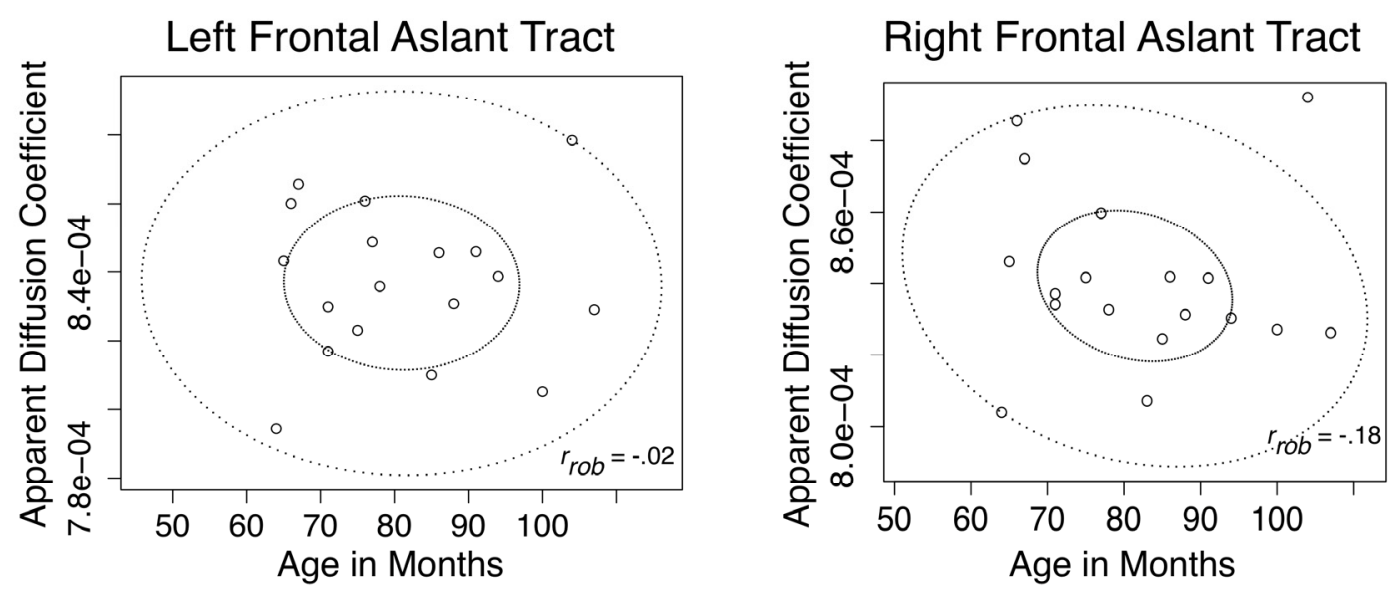

Left Superior Longitudinal

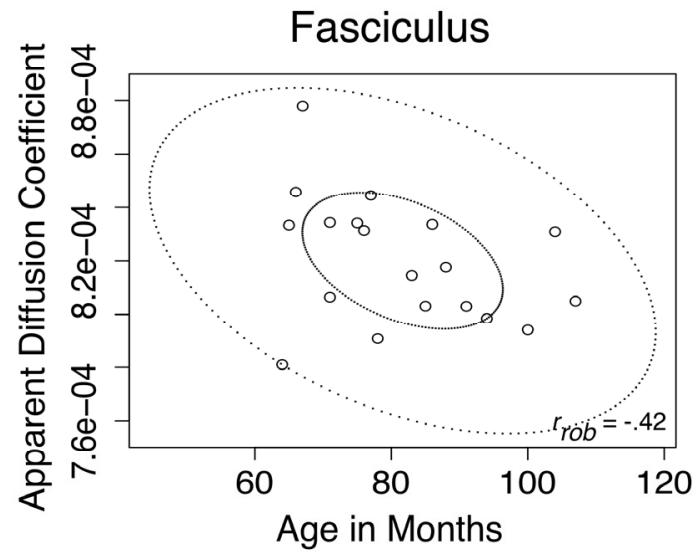

Right Superior Longitudinal

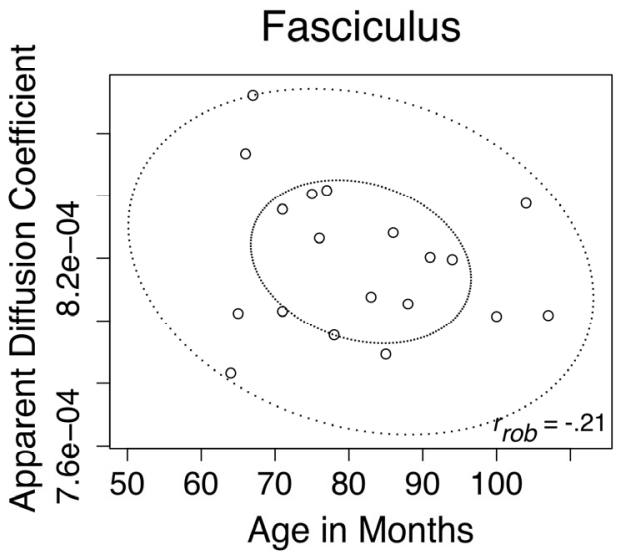

Figure 8. Top: Age-related changes in apparent diffusion coefficient in the left and right Frontal Aslant Tract. Bottom: Age-related changes in apparent diffusion coefficient in the left and right Superior Longitudinal Fasciculus/Arcuate Fasciculus.

\section{Relation of the FAT and SLF/AF to Speech, Language, and Literacy}

To relate the FAT and SLF/AF white matter integrity to speech, language, and literacy we conducted robust linear models. For these models we bootstrapped the residuals (10,000 iterations) to obtain the $95 \%$ confidence intervals. The effects are reported after controlling for age in months, non-verbal IQ, sex, and whole brain FA in 
the model. The adjusted $\mathrm{R}^{2}$ values are reported from the ordinary least squares model; these are not available for robust models, and should be interpreted with caution. In Table 1 on the next page, only those findings in which the bootstrapped confidence intervals do not cover zero are reported. Results indicate that the right FAT was negatively related to phonology. Therefore, increased white matter integrity of the right FAT as measured by FA values, was associated with lower phonological scores. Moreover, we failed to find evidence that the FAT white matter integrity (as measured by FA and ADC) was related to our measures of $\mathrm{AV}$ speech perception, articulation, language, or literacy. In contrast, we found that both the left and right SLF/AF were related to language, specifically receptive language, expressive vocabulary, and language content, as measured by the CELF 
Frontal Aslant Tract (FAT) and Superior longitudinal fasciculus/arcuate fasciculus integrity predicts receptive and expressive language and

phonology

\section{Fractional Anisotropy (FA) Measures}

Predictor $\rightarrow$ Outcome $\quad$ B (SE) $\quad B \quad 95 \%$ CI $\quad R_{\text {ad }}^{2}$

Right FAT $\rightarrow$ Phonology

$-186.3(88.8)$

$-0.46$

-360.2 to -12.0

0.16

Left SLF/AF $\rightarrow$ CELF Receptive Language

$547.0(131.5)$

0.66

287.1 to 802.7

0.51

Left SLF/AF $\rightarrow$ CELF Language Content

$484.4(120.4)$

0.60

247.5 to 719.6

0.64

Right SLF/AF $\rightarrow$ CELF Receptive Language

$396.4(118.2)$

0.52

168.9 to 632.4

0.30

Right SLF/AF $\rightarrow$ CELF Language Content

$265.2(113.3)$

0.36

43.4 to 487.7

0.46

Right SLF/AF $\rightarrow$ CELF Expressive Vocabulary

$66.9(31.4)$

$0.42 \quad 5.3$ to 128.6

0.12

$\underline{\text { Apparent Diffusion Coefficient (ADC) Measures }}$

Left SLF/AF $\rightarrow$ CELF Receptive Language

Left SLF/AF $\rightarrow$ CELF Language Content

Left SLF/AF $\rightarrow$ CELF Expressive Vocabulary

Right SLF/AF $\rightarrow$ CELF Receptive Language

Right SLF/AF $\rightarrow$ CELF Language Content
$-26.6(9.1)$

$-22.7(8.0)$

$-6.3(2.3)$

$-25.4(9.1)$

$-18.3(8.2)$
$-0.45$

$-0.40$

$-0.52$

$-0.43$

$-0.32$

-45.0 to -9.2

0.24

-39.0 to -7.4

0.54

-10.8 to -1.7

0.10

-43.2 to -7.5

0.46

Note. Effects reported for robust linear models after controlling for age in months, sex, and whole brain FA (for FA predictors) or whole brain ADC (for ADC predictors), and block design. FAT $=$ Frontal Aslant Tract; SLF/AF $=$ Superior longitudinal fasciculus/arcuate fasciculus. CELF $=$ Clinical Evaluation of Language Fundamentals. To reduce digits, $\mathrm{ADC}$ values were divided by a constant. Adjusted $\mathrm{R}^{2}$ values are reported from the ordinary least squares model. 


\section{CHAPTER VI}

\section{DISCUSSION}

The work presented here showed that different white matter pathways can be identified, tracked, characterized, and quantified in terms of their structural integrity, and these metrics can be related to a number of behavioral outcome measures. In the present study we identified a new fiber pathway — the frontal aslant tract (FAT) - for the first time in young children. In addition to the FAT, we also characterized the superior longitudinal fasciculus/arcuate fasciculus (SLF/AF), another pathway of the dorsal speech stream, and related the two tracts to speech, language, and literacy outcomes. The findings in the current study contribute to a growing understanding of brain development and its relation to behavior.

\section{Frontal Aslant Tract (FAT)}

The FAT is a newly described fiber tract that has been recently identified in adults (Catani et al., 2012; Ford, McGregor, Case, Crosson, \& White, 2010; Lawes et al., 2008; Oishi et al., 2008). However, this is the first study to identify the FAT in children. We were able to identify the FAT bilaterally in almost all children. Advances in our understanding of FAT connectivity is important because of its putative involvement in speech production, speech perception, language, and literacy, especially in children who are continuing to develop these skills.

The FAT has been suggested to make connections from the superior frontal gyrus (Lawes et al., 2008), the posterior SMA (Oishi et al., 2008), and the pre-SMA (Ford et al., 2010) to the inferior frontal gyrus (Catani et al., 2013). It has been suggested to play a role in speech production (Catani et al., 2013; Dick et al., 2013) and found to be related 
to verbal fluency in patients with primary progressive aphasia (Catani et al., 2013).

Several fMRI studies suggest that motor regions are activated during speech perception tasks (Skipper et al., 2005; Skipper et al., 2007; Tremblay \& Small, 2011; Wilson, 2004; Wilson \& Iacoboni, 2006). The shared neural activation maps between speech perception and production suggest a close relationship between sensory and motor domains that may be important for communicating information about the acoustic speech sounds (e.g., /ba/) and how to produce these sounds (Tremblay \& Small, 2011). The primary finding of this study was that the FAT indeed provides a direct connection between the posterior SMA (Oishi et al., 2008), and the pre-SMA (Ford et al., 2010) to the inferior frontal gyrus (Catani et al., 2013). However, the FAT did not show age-related change. Absence of age-related change in the FAT may reflect that the tract is rather mature by 5-8 years old. Furthermore, it may be that age-related changes could be observed at younger ages, for example, when infants are learning to babble.

In addition, we expected the FAT to play a critical role in speech and language, especially in children who are currently developing these skills. In the present study, we found a significant negative relationship between white matter integrity of the right FAT and phonology. This finding was unexpected. If any relationship existed, we expected both the left and right FAT to be positively related to phonology. However, the relation between FAT integrity and phonology may be better understood by further delineating different connections within the pathway. That is, evidence suggests that the pre-SMA and SMA are anatomically and functionally distinct, with the pre-SMA a prefrontal region and the SMA a non-primary motor region (Luppino, Matelli, Camarda, \& Rizzolatti, 1994; Picard \& Strick, 1996; Tremblay \& Gracco, 2010). Therefore, isolating 
pre-SMA and SMA might better capture interactions between white matter integrity and speech and language. In addition, fMRI studies have suggested the pre-SMA as playing a critical role in more complex/cognitive motor control (Kim et al., 2010), such as the selection of words and oral motor responses (Tremblay \& Gracco, 2010). Simultaneous activity in the pre-SMA and IFG has been reported as critical in the selection process of spoken word generation (e.g., compared to reading aloud; Tremblay \& Gracco, 2006). In comparison, the SMA has been implicated in making connections with the spinal cord and important in action generation (Tremblay \& Gracco, 2006). The aforementioned evidence suggests the possibility that there are two functionally distinct tracts, similar to the SLF/AF (discussed below), and the fiber tracts from the pre-SMA may be correlated with online selection and generation of speech, compared with the SMA in repetition of speech. Broadly, isolating contributions of the pre-SMA and SMA may more accurately characterize the relationship between the FAT and speech, language, and literacy.

In addition to the negative relationship between the right FAT and phonology, we also failed to find a significant relationship between the left and right FAT and AV speech perception, articulation, language, and literacy. While the non-significant relationship of the FAT and literacy was expected, we did expect a relationship between FAT integrity and articulation. In this case, it may be that the FAT is mature by the age range we tested, and shows little age-related change. Our behavioral measures of articulation suggest that typical developing children do not vary much in their ability produce speech sounds - most children performed well on the DEAP, with a range between 75 and 110, and the majority of children scoring above $105(n=15)$, a score of 110 indicating ceiling performance. Thus, one way of looking at this is that the 
behavioral and brain data are congruent and show relative maturity at this age. However, this result should be interpreted with caution because of the ceiling effects in the behavioral measures. Moreover, children in the present study are typically developing. Therefore, we might observe a different brain/behavior relation in children who have speech disorders (Sener, 2001). Future studies that investigate a younger age cohort, or children with speech pathologies, may reveal a fundamental relationship between this pathway and articulation.

\section{Superior Longitudinal Fasciculus/Arcuate Fasciculus (SLF/AF)}

The present study also characterized the SLF/AF bilaterally in all children. The SLF/AF has been well studied in both adults and children. Previous work with older children (7-11 years old) suggests that left AF fibers show age-related changes (Yeatman et al., 2011). Schmithorst and colleagues (2002) also report significant positive correlation of FA with age (5-18 years old) in the left arcuate fasciculus, but not the right. The lateralization of change in FA is similar to what we reported here. The present work also focuses on a narrow age range (5-8 years) because during this age range children are developing speech, language, and literacy skill. We expanded on these finding by focusing on contributions from both the left and right hemisphere and showing that the left and right hemispheres show a different developmental trend, with the left SLF/AF showing a significant increase in FA with age.

The current study found asymmetric trajectories of brain development in the SLF/AF. We found age-related changes in white matter integrity in left SLF/AF but not the right. The SLF/AF connects frontal, temporal, and parietal lobes (Hickok \& Poeppel, 2007), which are important for processing speech, language, and literacy (Paus et al., 
1999; Beaulieu et al., 2005; Klingberg et al., 2000). In addition, while both hemispheres are implicated in language processing, evidence suggests that more generally, language processing is strongly left dominant (Hickok \& Poeppel, 2007). Therefore, age-related changes in the left SLF/AF but not the right may reflect asymmetrical re-organization of the SLF/AF fiber pathway to be more coherent during language development. Of note, the work presented here also suggests that maturation of the SLF/AF is not the sole predictor of language. For example, despite that age-related changes in white matter integrity were specific to the left SLF/AF, we found that white matter integrity in both the left and right SLF/AF predicted receptive language, expressive vocabulary, and language content. These results were robust even after controlling for age, sex, non-verbal IQ, and whole brain white matter integrity.

Moreover, we found a non-significant relationship between white matter integrity of the SLF/AF and speech and literacy. Other studies have provided evidence for white matter integrity in the SLF/AF for reading (Klingberg et al., 2000), phonological awareness (Yeatman et al., 2011), and speech (Catani et al., 2005). However, aside from Yeatman and colleagues (2011), the aforementioned studies were conducted in adults. The distinct findings may also be related to evidence suggesting that different components of the SLF/AF contribute uniquely to speech (Catani et al., 2005), and literacy (Thiebaut de Schotten et al., 2012). Therefore, the next step should be to characterize the different components of the SLF/AF, for the same reasons proposed for the FAT, and relate them to speech and literacy. 


\section{Importance of Findings for a Model of the Neurobiology of Language}

More broadly, the work presented here complements the dorsal-ventral-motor model for language. In the introduction, we proposed the SLF/AF and the FAT as important for language. We characterized the SLF/AF in the developing brain and found that both the left and right SLF/AF predicted receptive language, expressive vocabulary, language content, but not speech and literacy. In retrospect, this makes sense because our measures of receptive and expressive language are based on the child's listening and auditory comprehension abilities and their ability to name illustrations of people, objects, and actions, respectively. Successfully doing this task requires the entire SLF/AF tract and both hemispheres.

The primary finding was that both the left and right SLF/AF predicted language, specifically receptive and expressive language, and language content. While both the left and right SLF/AF predicted receptive language and language content, the left SLF/AF as predictors accounted for more variance in the model compared with the right SLF/AF predictors with respect to receptive language and language content. The finding is consistent with models of language, which suggest that language is processed bilaterally, although not identically (Hickok \& Poeppel, 2004; Ivry, 1998; Poeppel, 2003; Zatorre, Meyer, Gjedde, \& Evans, 1996). That is, while the two hemispheres contribute to language processing, each hemisphere performs different computations on the incoming speech signal (Hickok, 2009). Thus, our findings suggest that the left and right SLF/AF are important for language, but their contributions are not identical.

We also found that the left SLF/AF and the right SLF/AF predicted expressive vocabulary, measured by the CELF. The expressive vocabulary subtest evaluates the 
student's ability to name illustrations of people, objects, and actions (referential naming). Both the left and right hemispheres are implicated in phonological processing and involve both motor/premotor cortices (Hickok \& Poeppel, 2007). Therefore, this finding is consistent with the idea that language is a bilateral process in the normal brain.

In addition, evidence from patients with neurological deficits reveals similar reports with respect to bilateral contributions to language processing. For example, auditory comprehension at the lexical level in the right hemisphere is spared in split-brain patients and patients undergoing the Wada test (Barde, Baynes, Gage, \& Hickok, 2000; McGlone, 1984). It is noteworthy, however, that findings from the present study do not negate that in typical right-handed individuals the left hemisphere is a prominent contributor to language function. Rather, the findings support the right hemisphere, more specifically the right SLF/AF, in language processing. In addition, while this study does not inform us about how different components of the SLF/AF participate in comprehension and articulation, it does reveal that the SLF/AF as a whole is important for language.

Lastly, the dorsal-ventral-motor model for language processing suggests a motor component. The FAT was suggested to make connection from typically motor regions to the IFG (Catani et al., 2013; Dick et al., 2013). Although the present study found no agerelated change or a functional specialization of the FAT with respect to speech, language, and literacy, this does not mean that the FAT does not serve an important function for language. In fact, as mentioned earlier, others have found the FAT important for language production (Catani et al., 2013). Incorporating the FAT as part of the dorsal-ventralmotor model for language is essential not only in understanding language, but also in 
other domains, for example, during neurosurgery, where avoiding fibers that are important for language and motor function are essential. Therefore, in addition to mapping prominent fiber tracts such as the SLF/AF and others from the ventral stream, as specified in the introduction, the FAT should also be considered.

\section{Summary}

In the present study, we were able to track the FAT for the first time in children. While the FAT did not show age-related change, the left SLF/AF did show age-related change, but the right SLF/AF did not. White matter integrity of the right FAT predicted phonology but did not predict performance on AV speech, articulation, language, and literacy. White matter integrity in the left and right SLF/AF predicted language, specifically receptive language, expressive vocabulary, and language content. Neither the left or right SLF/AF predicted speech or literacy. We propose understanding the relationship between the FAT and SLF/AF and speech and literacy requires isolating components from each that are implicated in speech and literacy. 


\section{LIST OF REFERENCES}

Aboitiz, F., Scheibel, A. B., Fisher, R. S., \& Zaidel, E. (1992). Individual differences in brain asymmetries and fiber composition in the human corpus callosum. Brain Research, 598(1), 154-161.

Arnal, L. H., Morillon, B., Kell, C. A., \& Giraud, A. L. (2009). Dual neural routing of visual facilitation in speech processing. The Journal of Neuroscience: The Official Journal of the Society for Neuroscience, 29(43), 13445-53. doi:10.1523/JNEUROSCI.3194-09.2009

Bahrick, L. E. (2010). Intermodal perception and selective attention to intersensory redundancy: Implications for typical social development and autism. Blackwell Handbook of Infant Development, 1, 120-165.

Bahrick, L. E., \& Lickliter, R. (2004). Infants' perception of rhythm and tempo in unimodal and multimodal stimulation: A developmental test of the intersensory redundancy hypothesis. Cognitive, Affective, \& Behavioral Neuroscience, 4(2), 137-147.

Bahrick, L. E., Lickliter, R., \& Flom, R. (2004). Intersensory redundancy guides the development of selective attention, perception, and cognition in infancy. Current Directions in Psychological Science, 13(3), 99-102. doi:10.1111/j.09637214.2004.00283.x

Barnea-Goraly, N., Menon, V., Eckert, M., Tamm, L., Bammer, R., Karchemskiy, A., . . . Reiss, A. L. (2005a). White matter development during childhood and adolescence: A cross-sectional diffusion tensor imaging study. Cerebral Cortex (New York, N.Y. : 1991), 15(12), 1848-54. doi:10.1093/cercor/bhi062

Barnea-Goraly, N., Menon, V., Eckert, M., Tamm, L., Bammer, R., Karchemskiy, A., . . . Reiss, A. L. (2005b). White matter development during childhood and adolescence: A cross-sectional diffusion tensor imaging study. Cerebral Cortex (New York, N.Y.: 1991), 15(12), 1848-54. doi:10.1093/cercor/bhi062

Barrós-Loscertales, A., Ventura-Campos, N., Visser, M., Alsius, A., Pallier, C., Avila Rivera, C., \& Soto-Faraco, S. (2013). Neural correlates of audiovisual speech processing in a second language. Brain and Language, 126(3), 253-262. doi:10.1016/j.bandl.2013.05.009

Basser, Mattiello, J., \& LeBihan, D. (1994). Estimation of the effective self-diffusion tensor from the NMR spin echo. Journal of Magnetic Resonance, 103, 247-254.

Bates, E., \& Dick, F. (2002). Language, gesture, and the developing brain. Developmental Psychobiology, 40(3), 293-310. doi:10.1002/dev.10034 
Bates, E., Benigni, L., Bretherton, I., Camaioni, L., \& Volterra, V. (1979). Cognition and communication from 9-13 months: Correlational findings. The Emergence of Symbols: Cognition and Communication in Infancy, 96-138.

Bauer, P. J., \& Thal, D. J. (1990). Scripts or scraps: Reconsidering the development of sequential understanding. Journal of Experimental Child Psychology, 50(2), 287304.

Bauer, P. J., Wenner, J. A., Dropik, P. L., \& Wewerka, S. S. (2000). Parameters of remembering and forgetting in the transition from infancy to early childhood. Monographs of the Society for Research in Child Development.

Beaulieu, C., Plewes, C., Paulson, L. A., Roy, D., Snook, L., Concha, L., \& Phillips, L. (2005). Imaging brain connectivity in children with diverse reading ability. NeuroImage, 25(4), 1266-1271.

Bernal, B., \& Altman, N. (2010). The connectivity of the superior longitudinal fasciculus: A tractography DTI study. Magn Reson Imaging, 28(2), 217-25. doi:10.1016

Blomert, L., \& Froyen, D. (2010). Multi-sensory learning and learning to read. International Journal of Psychophysiology : Official Journal of the International Organization of Psychophysiology, 77(3), 195-204. doi:10.1016/j.ijpsycho.2010.06.025

Breier, J. I., Hasan, K. M., Zhang, W., Men, D., \& Papanicolaou, A. C. (2008). Language dysfunction after stroke and damage to white matter tracts evaluated using diffusion tensor imaging. American Journal of Neuroradiology, 29(3), 483-487.

Buchsbaum, B. R., Hickok, \& Humphries, C. (2001). Role of left posterior superior temporal gyrus in phonological processing for speech perception and production. Cognitive Science, 25, 663-678.

Callan, D. E., Jones, J. A., Callan, A. M., \& Akahane-Yamada, R. (2004). Phonetic perceptual identification by native-and second-language speakers differentially activates brain regions involved with acoustic phonetic processing and those involved with articulatory--auditory/orosensory internal models. NeuroImage, 22(3), 1182-1194.

Calvert, . A. (1997). Activation of auditory cortex during silent lipreading. Science, 276(5312), 593-596. doi:10.1126/science.276.5312.593

Calvert, G. A., Campbell, R., \& Brammer, M. J. (2000). Evidence from functional magnetic resonance imaging of crossmodal binding in the human heteromodal cortex. Curr Biol, 10(11), 649-57. Retrieved from PubMed 
Catani, M., \& Mesulam, M. (2008). The arcuate fasciculus and the disconnection theme in language and aphasia: History and current state. Cortex, 44(8), 953-61. doi:10.1016/j.cortex.2008.04.002

Catani, M., DellAcqua, F., Vergani, F., Malik, F., Hodge, H., Roy, P., . . Thiebaut de Schotten, M. (2012). Short frontal lobe connections of the human brain. Cortex, $48(2), 273-291$.

Catani, M., Jones, D. K., \& ffytche, D. H. (2005). Perisylvian language networks of the human brain. Annals of Neurology, 57(1), 8-16. doi:10.1002/ana.20319

Catani, M., Mesulam, M. M., Jakobsen, E., Malik, F., Martersteck, A., Wieneke, C., .. . Weintraub, S. (2013). A novel frontal pathway underlies verbal fluency in primary progressive aphasia. Brain : A Journal of Neurology, 136(8), 2619-2628.

Coremans, J., Luypaert, R., Verhelle, F., Stadnik, T., \& Osteaux, M. (1994). A method for myelin fiber orientation mapping using diffusion-weighted MR images. Magnetic Resonance Imaging, 12(3), 443-454.

Coupé, P., Manjón, J. V., Robles, M., \& Collins, L. D. (2011). Adaptive multiresolution non-local means filter for 3D MR image denoising. IET Image Processing.

Descoteaux, M., Wiest-Daesslé, N., Prima, S., Barillot, C., \& Deriche, R. (2008). Impact of rician adapted non-local means filtering on HARDI. In Medical image computing and computer-assisted intervention - MICCAI 2008 (Vol. 5242, pp. 122-130). Berlin, Heidelberg: Springer Berlin Heidelberg. doi:10.1007/978-3540-85990-1_15

Desjardins, R.'. N., Rogers, J., \& Werker, J. F. (1997). An exploration of why preschoolers perform differently than do adults in audiovisual speech perception tasks. Journal of Experimental Child Psychology, (66), 85-110.

Dick, A. S., \& Tremblay, P. (2012). Beyond the arcuate fasciculus: Consensus and controversy in the connectional anatomy of language. Brain : A Journal of Neurology, 135(Pt 12), 3529-50. doi:10.1093/brain/aws222

Dick, A. S., Bernal, B., \& Tremblay, P. (2013). The language connectome: New pathways, new concepts. Neuroscientist. doi:10.1177/1073858413513502

Dick, A. S., Solodkin, A., \& Small, S. L. (2010). Neural development of networks for audiovisual speech comprehension. Brain and Language, 114(2), 101-14. doi:10.1016

Dodd, B., Zhu, H., Crosbie, S., Holm, A., \& Ozanne, A. (2002). Diagnostic evaluation of articulation and phonology (DEAP). Psychology Corporation. 
Dronkers, N. F. (1996). A new brain region for coordinating speech articulation. Nature, 384(6605), 159-161.

Dubois, J., Dehaene-Lambertz, G., Perrin, M., Mangin, J. -F., Cointepas, Y., Duchesnay, E., . . . Hertz-Pannier, L. (2008). Asynchrony of the early maturation of white matter bundles in healthy infants: Quantitative landmarks revealed noninvasively by diffusion tensor imaging. Human Brain Mapping, 29(1), 14-27.

Durgunouglu, A. Y., Nagy, W. E., \& Hancin-Bhatt, B. J. (1993). Cross-language transfer of phonological awareness. Journal of Educational Psychology, 85(3), 453.

Efron, B. (1992). Jackknife-after-bootstrap standard errors and influence functions. Journal of the Royal Statistical Society. Series B (Methodological), 83-127.

Efron, B., \& Tibshirani, R. (1986). Bootstrap methods for standard errors, confidence intervals, and other measures of statistical accuracy. Statistical Science, 54-75.

Erdener, D., \& Burnham, D. (2013). The relationship between auditory--visual speech perception and language-specific speech perception at the onset of reading instruction in english-speaking children. Journal of Experimental Child Psychology, 116(2), 120-138.

Farrell, J. A., Landman, B. A., Jones, C. K., Smith, S. A., Prince, J. L., van Zij1, P. C., \& Mori, S. (2007). Effects of signal-to-noise ratio on the accuracy and reproducibility of diffusion tensor imaging-derived fractional anisotropy, mean diffusivity, and principal eigenvector measurements at 1.5T. Journal of Magnetic Resonance Imaging, 26(3), 756-767. doi:10.1002/jmri.21053

Flechsig, P. (1896). Gehirn und seele, S. 84. Veit \& Comp., Leipzig.

Ford, A., McGregor, K. M., Case, K., Crosson, B., \& White, K. D. (2010). Structural connectivity of broca's area and medial frontal cortex. NeuroImage, 52(4), 12301237. doi:10.1016/j.neuroimage.2010.05.018

Geng, X., Gouttard, S., Sharma, A., Gu, H., Styner, M., Lin, W., . . Gilmore, J. H. (2012). Quantitative tract-based white matter development from birth to age 2years. NeuroImage, 61(3), 542-557.

Glasser, M. F., \& Rilling, J. K. (2008). DTI tractography of the human brain's language pathways. Cerebral Cortex (New York, N.Y. : 1991), 18(11), 2471-82. doi:10.1093/cercor/bhn011

Gogate, L. J., \& Bahrick, L. E. (1998). Intersensory redundancy facilitates learning of arbitrary relations between vowel sounds and objects in seven-month-old infants. Journal of Experimental Child Psychology, 69(2), 133-149. 
Gogate, L. J., Bahrick, L. E., \& Watson. (2000). A study of multimodal motherese: The role of temporal synchrony between verbal labels and gestures. Child Development, 71, 878-894.

Goldberg, K. M., \& Iglewicz, B. (1992). Bivariate extensions of the boxplot. Technometrics, 34(3), 307. doi:10.2307/1270037

Griffiths, T. D., \& Warren, J. D. (2002). The planum temporale as a computational hun. Trends in Neurosciences, 25(7), 348-353.

Hermoye, L., Saint-Martin, C., Cosnard, G., Lee, S. -K., Kim, J., Nassogne, M. -C., . . . Hua, K. (2006). Pediatric diffusion tensor imaging: Normal database and observation of the white matter maturation in early childhood. NeuroImage, 29(2), 493-504.

Hickok, G. (2009). The functional neuroanatomy of language. Phys Life Rev, 6(3), 12143. doi:10.1016/j.plrev.2009.06.001

Hickok, G., \& Poeppel, D. (2000). Towards a functional neuroanatomy of speech perception. Trends in Cognitive Sciences, 4(4), 131-138. Retrieved from PubMed.

Hickok, G., \& Poeppel, D. (2004). Dorsal and ventral streams: A framework for understanding aspects of the functional anatomy of language. Cognition, 92(1-2), 67-99. doi:10.1016/j.cognition.2003.10.011

Hickok, G., \& Poeppel, D. (2007). The cortical organization of speech processing. Nat Rev Neurosci, 8(5), 393-402. doi:10.1038/nrn2113

Jiang, H., van Zijl, P. C., Kim, J., Pearlson, G. D., \& Mori, S. (2006). DtiStudio: Resource program for diffusion tensor computation and fiber bundle tracking. Computer Methods and Programs in Biomedicine, 81(2), 106-16. doi:10.1016

Karbe, H., Herholz, K., Weber-Luxenburger, G., Ghaemi, M., \& Heiss, M. G. (1998). Cerebral networks and functional brain asymmetry: Evidence from regional metabolic changes during word repetition . Brain and Language, 63, 108-121.

Kasprian, G., Brugger, P. C., Weber, M., Krssák, M., Krampl, E., Herold, C., \& Prayer, D. (2008). In utero tractography of fetal white matter development. NeuroImage, $43(2), 213-224$.

Kim, J. -H., Lee, J. -M., Jo, H. J., Kim, S. H., Lee, J. H., Kim, S. T., . . Kim, S. I. (2010). Defining functional SMA and pre-sma subregions in human MFC using resting state fmri: Functional connectivity-based parcellation method. NeuroImage, 49(3), 2375-2386. 
Klingberg, T., Hedehus, M., Temple, E., Salz, T., Gabrieli, J. D., Moseley, M. E., \& Poldrack, R. A. (2000). Microstructure of temporo-parietal white matter as a basis for reading ability: Evidence from diffusion tensor magnetic resonance imaging. Neuron, 25(2), 493-500.

Klingberg, T., Vaidya, C. J., Gabrieli, J. D., Moseley, M. E., \& Hedehus, M. (1999). Myelination and organization of the frontal white matter in children: A diffusion tensor MRI study. Neuroreport, 10(13), 2817-2821.

Kuhl, P. K., \& Meltzoff, A. N. (1982). The bimodal perception of speech in infancy. In

Lachs, L., Pisoni, D. B., \& Iler Kirk, K. (2001). Use of audiovisual information in speech perception by prelingually deaf children with cochlear implants: A first report. Ear and Hearing, 22(3), 236-251.

Lawes, I. N. C., Barrick, T. R., Murugam, V., Spierings, N., Evans, D. R., Song, M., \& Clark, C. A. (2008). Atlas-based segmentation of white matter tracts of the human brain using diffusion tensor tractography and comparison with classical dissection. NeuroImage, 39(1), 62-79.

Lebel, C., Gee, M., Camicioli, R., Wieler, M., Martin, W., \& Beaulieu, C. (2012). Diffusion tensor imaging of white matter tract evolution over the lifespan. NeuroImage, 60(1), 340-352.

Lenroot, R. K., \& Giedd, J. N. (2006). Brain development in children and adolescents: Insights from anatomical magnetic resonance imaging. Neuroscience \& Biobehavioral Reviews, 30(6), 718-729. Retrieved from Google Scholar.

Lewedag, V. L., Oller, D. K., \& Lynch, M. P. (1994). Infants' vocalization patterns across home and laboratory environments. First Language, 14(42-43), 049-65.

Liberman, I., \& Shankweiler, D. (1987). Phonology and the problems of learning to read and write. Advances in Learning and Behavioral Disabilities. Greenwich, CT: Jai Press.

Lundberg, I., Olofsson, \& Wall, S. (1980). Reading and spelling skills in the first school years predicted from phonemic awareness skills in kindergarten. Scandinavian Journal of Psychology, 21(1), 159-173.

Luppino, G., Matelli, M., Camarda, R., \& Rizzolatti, G. (1994). Corticospinal projections from mesial frontal and cingulate areas in the monkey. Neuroreport, 5(18), 25452548 
Mabbott, D. J., Noseworthy, M., Bouffet, E., Laughlin, S., \& Rockel, C. (2006). White matter growth as a mechanism of cognitive development in children. NeuroImage, 33(3), 936-946.

Magnan, A., \& Ecalle, J. (2006). Audio-visual training in children with reading disabilities. Computers \& Education, 46(4), 407-425. doi:10.1016

Magnan, A., Ecalle, J., Veuillet, E., \& Collet, L. (2004). The effects of an audio-visual training program in dyslexic children. Dyslexia, 10(2), 131-140.

Martino, J., De Witt Hamer, P. C., Berger, M. S., Lawton, M. T., Arnold, C. M., de Lucas, E. M., \& Duffau, H. (2013). Analysis of the subcomponents and cortical terminations of the perisylvian superior longitudinal fasciculus: A fiber dissection and DTI tractography study. Brain Structure \& Function, 218(1), 105-21. doi:10.1007/s00429-012-0386-5

Masataka, N. (2001). Why early linguistic milestones are delayed in children with williams syndrome: Late onset of hand banging as a possible rate--limiting constraint on the emergence of canonical babbling. Developmental Science, 4(2), $158-164$.

Massaro, D. W., Thompson, L. A., Barron, B., \& Laren, E. (1986). Developmental changes in visual and auditory contributions to speech perception. Journal of Experimental Child Psychology, 41(1), 93-113.

Mattingly, I. G. (1972). Reading, the linguistic process, and linguistic awareness. Massachusetts Inst. of Technology P. Retrieved from Common.

McGraw, P., Liang, L., \& Provenzale, J. M. (2002). Evaluation of normal age-related changes in anisotropy during infancy and childhood as shown by diffusion tensor imaging. American Journal of Roentgenology, 179(6), 1515-1522.

McGurk, H., \& MacDonald, J. (1976). Hearing lips and seeing voices.

Miller, L. M., \& D'Esposito, M. (2005). Perceptual fusion and stimulus coincidence in the cross-modal integration of speech. The Journal of Neuroscience : The Official Journal of the Society for Neuroscience, 25(25), 5884-93. doi:10.1523/JNEUROSCI.0896-05.2005

Mori, S., \& Zhang, J. (2006). Principles of diffusion tensor imaging and its applications to basic neuroscience research. Neuron, 51(5), 527-539.

Morriss, M. C., Zimmerman, R. A., Bilaniuk, L. T., Hunter, J. V., \& Haselgrove, J. C. (1999). Changes in brain water diffusion during childhood. Neuroradiology, 41(12), 929-934. 
Nagy, Z., Westerberg, H., \& Klingberg, T. (2004). Maturation of white matter is associated with the development of cognitive functions during childhood. Journal of Cognitive Neuroscience, 16(7), 1227-1233.

Nath, A. R., \& Beauchamp, M. S. (2012). A neural basis for interindividual differences in the mcgurk effect, a multisensory speech illusion. NeuroImage, 59(1), 781-7. doi:10.1016/j.neuroimage.2011.07.024

Nath, A. R., Fava, E. E., \& Beauchamp, M. S. (2011). Neural correlates of interindividual differences in children's audiovisual speech perception. The Journal of Neuroscience: The Official Journal of the Society for Neuroscience, 31(39), 13963-71. doi:10.1523/JNEUROSCI.2605-11.2011

Oishi, K., Zilles, K., Amunts, K., Faria, A., Jiang, H., Li, X., . . Toga, A. W. (2008). Human brain white matter atlas: Identification and assignment of common anatomical structures in superficial white matter. NeuroImage, 43(3), 447-457.

Okada, K., \& Hickok, G. (2009). Two cortical mechanisms support the integration of visual and auditory speech: A hypothesis and preliminary data. Neurosci Lett, 452(3), 219-23. doi:10.1016/j.neulet.2009.01.060

Paus, T., Collins, D. L., Evans, A. C., Leonard, G., Pike, B., \& Zijdenbos, A. (2001). Maturation of white matter in the human brain: A review of magnetic resonance studies. Brain Research Bulletin, 54(3), 255-266.

Paus, T., Zijdenbos, A., Worsley, K., Collins, D. L., Blumenthal, J., Giedd, J. N., . . . Evans, A. C. (1999). Structural maturation of neural pathways in children and adolescents: In vivo study. Science, 283(5409), 1908-1911.

Picard, N., \& Strick, P. L. (1996). Motor areas of the medial wall: A review of their location and functional activation. Cerebral Cortex, 6(3), 342-353.

Poeppel, D., Emmorey, K., Hickok, G., \& Pylkkänen, L. (2012). Towards a new neurobiology of language. The Journal of Neuroscience, 32(41), 14125-14131.

Qiu, D., Tan, L. H., Zhou, K., \& Khong, P. L. (2008). Diffusion tensor imaging of normal white matter maturation from late childhood to young adulthood: Voxel-wise evaluation of mean diffusivity, fractional anisotropy, radial and axial diffusivities, and correlation with reading development. NeuroImage, 41(2), 223-32. doi:10.1016/j.neuroimage.2008.02.023

Read, C., Yun-Fei, Z., Hong-Yin, N., \& Bao-Qing, D. (1986). The ability to manipulate speech sounds depends on knowing alphabetic writing. Cognition, 24(1), 31-44. 
Reiss, A. L., Abrams, M. T., Singer, H. S., Ross, J. L., \& Denckla, M. B. (1996). Brain development, gender and IQ in children A volumetric imaging study. Brain : A Journal of Neurology, 119(5), 1763-1774.

Ross, L. A., Molholm, S., Blanco, D., Gomez-Ramirez, M., Saint-Amour, D., \& Foxe, J. J. (2011). The development of multisensory speech perception continues into the late childhood years. The European Journal of Neuroscience, 33(12), 2329-37. doi:10.1111/j.1460-9568.2011.07685.x

Schmithorst, V. J., Holland, S. K., \& Plante, E. (2011). Diffusion tensor imaging reveals white matter microstructure correlations with auditory processing ability. Ear and Hearing, 32(2), 156.

Schmithorst, V. J., Wilke, M., Dardzinski, B. J., \& Holland, S. K. (2002). Correlation of white matter diffusivity and anisotropy with age during childhood and adolescence: A cross-sectional diffusion-tensor MR imaging study. Radiology, $222(1), 212$.

Schmithorst, V. J., Wilke, M., Dardzinski, B. J., \& Holland, S. K. (2005). Cognitive functions correlate with white matter architecture in a normal pediatric population: A diffusion tensor MRI study. Human Brain Mapping, 26(2), 139147.

Schneider, J. F. L., Ilyasov, K. A., Hennig, J., \& Martin, E. (2004). Fast quantitative diffusion-tensor imaging of cerebral white matter from the neonatal period to adolescence. Neuroradiology, 46(4), 258-266.

Schrank, F. A., \& Mather, N. (2006). Woodcock-Johnson III (r) diagnostic reading battery. Rolling Meadows, IL: Riverside.

Sekiyama, K., \& Burnham, D. (2004). Issues in the development of auditory-visual speech perception: Adults, infants, and children. INTERSPEECH.

Sekiyama, K., \& Burnham, D. (2008). Impact of language on development of auditoryvisual speech perception. Dev Sci, 11(2), 306-20. doi:10.1111/j.1467-

7687.2008.00677.x

Sener, R. N. (2001). Diffusion MRI: Apparent diffusion coefficient (ADC) values in the normal brain and a classification of brain disorders based on ADC values. Computerized Medical Imaging and Graphics, 25(4), 299-326.

Semel-Mintz, E., Wiig, E., \& Secord, W. (2003). Clinical evaluation of language fundamentals (4th ed). San Antonio, TX., Psychological Corporation. 
Shalom, D. B., \& Poeppel, D. (2008). Functional anatomic models of language: Assembling the pieces. The Neuroscientist, 14(1), 119-127.

Shaywitz, S. E., \& Shaywitz, B. A. (2008). Paying attention to reading: The neurobiology of reading and dyslexia. Development and Psychopathology, 20(4), 1329-49. doi:10.1017/S0954579408000631

Shore, C., Bates, E., Bretherton, I., Beeghly, M., \& OConnell, B. (1990). Vocal and gestural symbols: Similarities and differences from 13 to 28 months. In From gesture to language in hearing and deaf children (pp. 79-91). Springer.

Skipper, J. I., Nusbaum, H. C., \& Small, S. L. (2005). Listening to talking faces: Motor cortical activation during speech perception. NeuroImage, 25(1), 76-89. doi:10.1016/j.neuroimage.2004.11.006

Skipper, J. I., van Wassenhove, V., Nusbaum, H. C., \& Small, S. L. (2007). Hearing lips and seeing voices: How cortical areas supporting speech production mediate audiovisual speech perception. Cerebral Cortex (New York, N.Y. : 1991), 17(10), 2387-99. doi:10.1093/cercor/bhl147

Temple, E., Deutsch, G. K., Poldrack, R. A., Miller, S. L., Tallal, P., Merzenich, M. M., \& Gabrieli. (2003). Neural deficits in children with dyslexia ameliorated by behavioral remediation: Evidence from functional MRI. Proceedings of the National Academy of Sciences, 100.5, 2860-2865.

Temple, E., Poldrack, R. A., Salidis, J., Deutsch, G. K., Tallal, P., Merzenich, M. M., \& Gabrieli, J. D. E. (2001). Disrupted neural responses to phonological and orthographic processing in dyslexic children: An fmri study. Brain Imaging, 12(2), 299-307.

Thiebaut de Schotten, M., Cohen, L., Amemiya, E., Braga, L. W., \& Dehaene, S. (2012). Learning to read improves the structure of the arcuate fasciculus. Cerebral Cortex (New York, N.Y. : 1991). doi:10.1093/cercor/bhs383

Thiebaut de Schotten, M., Dell'Acqua, F., Valabregue, R., \& Catani, M. (2012). Monkey to human comparative anatomy of the frontal lobe association tracts. Cortex, 48(1), 82-96. doi:10.1016/j.cortex.2011.10.001

Tremblay, P., \& Gracco, V. L. (2006). Contribution of the frontal lobe to externally and internally specified verbal responses: FMRI evidence. NeuroImage, 33(3), 947957.

Tremblay, P., \& Gracco, V. L. (2010). On the selection of words and oral motor responses: Evidence of a response-independent fronto-parietal network. Cortex, 46(1), 15-28. 
Tremblay, P., \& Small, S. L. (2011). On the context-dependent nature of the contribution of the ventral premotor cortex to speech perception. NeuroImage, 57(4), 1561-71. doi:10.1016/j.neuroimage.2011.05.067

Van Linden, S., \& Vroomen, J. (2008). Audiovisual speech recalibration in children*. Journal of Child Language, 35(4), 809.

van Wassenhove, V., Grant, K. G., \& Poeppel. (2005). Visual speech speeds up the neural processing of auditory speech. PNAS, 102(4), 1181-1186.

Wagner, R. K., Torgesen, J. K., Rashotte, C. A., Hecht, S. A., Barker, T. A., Burgess, S. R., ... Garon, T. (1997). Changing relations between phonological processing abilities and word-level reading as children develop from beginning to skilled readers: A 5-year longitudinal study. Developmental Psychology, 33(3), 468-479.

Watkins, K. E., Strafella, A. P., \& Paus, T. (2003). Seeing and hearing speech excites the motor system involved in speech production. Neuropsychologia, 41(8), 989-94. Retrieved from PubMed.

Wechsler, D. (2002). Wechsler preschool and primary scale of intelligencetm third edition (WPPSITM-III). Sydney, NSW: Pearson.

Wiest-Daesslé, N., Prima, S., Coupé, P., Morrissey, S. P., \& Barillot, C. (2008). Rician noise removal by non-local means filtering for low signal-to-noise ratio MRI: Applications to DT-MRI. MICCAI, 2, 171-179.

Wilcox, R. R. (2012). Introduction to robust estimation and hypothesis testing. Academic Press.

Wilke, M., Krägeloh-Mann, I., \& Holland, S. K. (2007). Global and local development of gray and white matter volume in normal children and adolescents. Experimental Brain Research, 178(3), 296-307.

Wilson, S. M. (2004a). Listening to speech activates motor areas involved in speech production. Nature Neuroscience, 7(7), 701-702.

Wilson, S. M. (2004b). Listening to speech activates motor areas involved in speech production. Nature Neuroscience, 7(7), 701-702.

Wilson, S. M., \& Iacoboni, M. (2006). Neural responses to non-native phonemes varying in producibility: Evidence for the sensorimotor nature of speech perception. NeuroImage, 33(1), 316-325.

Wright, D. B., \& London, K. (2009). Modern regression techniques using $R$ : A practical guide. Sage. 
Yeatman, J. D., Dougherty, R. F., Rykhlevskaia, E., Sherbondy, A. J., Deutsch, G. K., Wandell, B. A., \& Ben-Shachar, M. (2011). Anatomical properties of the arcuate fasciculus predict phonological and reading skills in children. J Cogn Neurosci, 23(11), 3304-17. doi:10.1162/jocn_a_00061

Zhai, G., Lin, W., Wilber, K. P., Gerig, G., \& Gilmore, J. H. (2003). Comparisons of regional white matter diffusion in healthy neonates and adults performed with a 3.0-T head-only MR imaging unit1. Radiology, 229(3), 673-681.

Zhang, S., Demiralp, C., \& Laidlaw, D. H. (2003). Visualizing diffusion tensor MR images using streamtubes and streamsurfaces. Visualization and Computer Graphics, IEEE Transactions on, 9(4), 454-462. 